\title{
An Improved Memory State Feedback RMPC for Uncertain Constrained Linear Systems with Multiple Uncertain Delays
}

\author{
Weiwei Qin, ${ }^{1}$ Bing He, ${ }^{1}$ Pengtao Zhao, ${ }^{2}$ and Gang Liu ${ }^{1}$ \\ ${ }^{1}$ Department of Aerospace Engineering, Xian Research Institute of High Technology, Xian 710025, China \\ ${ }^{2}$ College of Science, Xian Research Institute of High Technology, Xian 710025, China \\ Correspondence should be addressed to Weiwei Qin; qww_1982@163.com
}

Received 4 November 2014; Accepted 30 December 2014

Academic Editor: Zhiguang Feng

Copyright (c) 2015 Weiwei Qin et al. This is an open access article distributed under the Creative Commons Attribution License, which permits unrestricted use, distribution, and reproduction in any medium, provided the original work is properly cited.

\begin{abstract}
The problem of robust asymptotic stabilization is considered for a class of discrete-time uncertain linear systems with multiple uncertain time-delayed states and input constraints. Compared with other works in the literature, the proposed approach takes the information of the delayed states with the estimated time-delays indices into full consideration. Based on the predictive control principle of receding horizon optimization and Lyapunov stability theory combined with linear matrix inequalities (LMIs) techniques, a time-delayed state dependent quadratic function is considered for incorporating MPC problem formulation. The robust MPC problem is formulated to minimize the upper bound of infinite horizon cost that satisfies the sufficient conditions. The proposed approach allows for the synthesis of robust memory state feedback controllers with respect to uncertainties on the implemented delay. Since developing the improved memory state feedback controller, the novel improved method is much less conservative and more general. Finally, the numerical simulation results prove availability of the proposed method.
\end{abstract}

\section{Introduction}

Model predictive control (MPC), also known as receding horizon control, is a mature technology and has become the standard approach for implementing constrained, multivariable control in the process industries today. MPC provides an integrated solution for controlling systems with interacting variables, complex dynamics, and constraints [1-3]. The basic concept of MPC is to solve an open-loop constrained optimization problem at each time instant and implement only the first control move of the solution. This procedure is repeated at the next time instant.

It is well known that parameter uncertainties and timedelays cannot be avoided in practice, especially in many chemical processes where the MPC has been mainly applied. Since the parameter uncertainties and time-delays are frequently the main cause of performance degradation and instability, there has been an increasing interest in the robust control of uncertain time-delay systems in the control literature [4]. Recently, many researchers have focused on the robust MPC of time-delay systems but only a few MPC algorithms have been developed to explicitly handle timedelayed systems [4-17].

The authors designed the novel robust MPC for uncertain systems and argued that the control scheme can be extended to the time-delayed systems [4]. But if the delay indices are unknown, it is not straightforward and not easy to show the feasibility of the online optimization problem and guarantee the closed-loop stability. Kwon et al. [5] proposed a simple receding horizon controller for continuous time systems with state delay, where the reduction technique was adopted so that an optimal problem for state delayed systems could be transformed into an optimal problem for delay free ordinary systems. But the method cannot guarantee the stability. A memory state feedback RMPC for uncertain linear systems with multiple known time-delayed states was proposed in [7]. Furthermore, an MPC algorithm for uncertain timevarying systems with input constraints and state-delay was proposed in [8], and the delay was assumed unknown but with a known upper bound. However, the results are rather conservative because feedback controller consists of only current state. The robust controllers called memory state 
feedback controller were proposed for uncertain systems with time-delay state and input constraints in $[12,14]$. The proposed methods were derived from the minimization problem of infinite horizon cost by adopting memory state feedback with a delay index minimizing state estimation errors. Furthermore, a $\delta$-memory-resilient controller was addressed for uncertain LTI/LPV time-delay systems with respect to an uncertain knowledge of the implemented delay in [15]. And a state feedback controller by developing a delay decomposition approach was presented for linear uncertain system with time-varying delay in [16]. But in practice there exist multiple time-delay states sometimes. However, only a few MPC methods handle systems with multiple unknown time-delayed states explicitly, which is the main topic of this paper. A robust MPC algorithm for a class of uncertain multiple state and input time-delay systems with nonlinear disturbance was proposed in [17]. But the results were also conservative because feedback controller consists of only current state.

In this section, we propose a novel delay-dependent RMPC algorithm for a class of linear uncertain systems with multiple uncertain time-delayed states and input constraints, by taking the information of the multiple delayed states with estimated time-delay indices into full consideration. For the unknown time-delayed states, we consider uncertainties of the estimated delay state knowledge and take it into account in an efficient way in the synthesis. Comparing with previous results [4], the proposed method allows an improvement in system performance in the sense of minimizing the upper bound of the infinite horizon cost. And comparing with the exact-memory method, the proposed method is more realistic, from a practical point of view.

The paper is organized as follows. In Section 2, the problem to be tackled is stated and some standard assumptions are introduced. Section 3 supplies a robust delay-dependent state feedback controller which is proposed for multiple uncertain time-delayed linear uncertain systems with input constraints. Section 4 minimizes the upper bound of infinite horizon cost concluding the estimated time-delay indices that satisfies the sufficient conditions, in terms of linear matrix inequality (LMI). Section 5 illustrates the performance of the proposed controller by means of an example. Finally, in Section 6, we make some concluding remarks.

\section{Problem Statement}

Consider the following class of discrete-time linear uncertain systems with multiple uncertain delayed states:

$$
\begin{aligned}
x(k+1)= & (A+\Delta A) x(k)+\sum_{\tau=1}^{l} A_{d \tau} x\left(k-d_{\tau}\right) \\
& +(B+\Delta B) u(k) \\
& x(k)=\phi(k) \quad-d^{*} \leq k \leq 0,
\end{aligned}
$$

where $x(k) \in \mathbb{R}^{n}, u(k) \in \mathbb{R}^{m}$, and $\phi(k) \in \mathbb{R}^{n}$ are the system state, control input, and the initial condition, respectively.
The system is subject to constraints on control actions, and they are given by

$$
-u_{\max } \leq u(k) \leq u_{\max }, \quad u_{\max }>0 \forall k \in[0, \infty)
$$

and $d_{\tau}>0(\tau=1, \ldots, l)$ denotes the uncertain delay, but being assumed to

$$
0 \leq d_{\tau, \min } \leq d_{\tau} \leq d_{\tau, \max } \leq d^{*}, \quad \tau=1, \ldots, l,
$$

with $0 \leq d_{1} \leq d_{2} \leq \cdots \leq d_{l} \leq d^{*}$ and the bounded value $d^{*}$ is a known integer, and $l$ is constant representing the number of delayed states. $A, A_{d 1}, \ldots, A_{d l}$ and $B$ are known constant matrices with appropriate dimensions.

The structured uncertainties $[\Delta A, \Delta B]$ are time-varying matrices with appropriate dimensions and are defined by

$$
[\Delta A, \Delta B]=H \cdot F(\cdot)\left[E_{1}, E_{2}\right],
$$

where $H, E_{1}$, and $E_{2}$ are known constant real matrices with appropriate dimensions. And $F(\cdot)$ is an unknown real and time-varying matrix with Lebesgue-measurable elements such that $F^{T}(\cdot) F(\cdot) \leq I$. It is assumed that system (1) is stabilizable for the existence of a stabilizing feedback control and that the state $x(k)$ is available at every sampling instant $k$.

Lemma 1 (see [18]). Given any vectors $Z, Y \in \mathbb{R}^{n}$ and any positive definite symmetric matrices $P=P^{T} \in \mathbb{R}^{n \times n}$,

$$
2 Z^{T} P Y \leq Z^{T} P Z+Y^{T} P Y .
$$

Lemma 2 (see [18]). Given matrices $A, D, E$, and $F$ with appropriate dimensions and for all $F$ satisfying $\|F\|<1$, if there exist some positive definite symmetric matrices $P=P^{T}>0$ and $\varepsilon>0$ such that $P-\varepsilon D D^{T}>0$, then

$$
(A+D F E)^{T} P^{-1}(A+D F E)<A^{T}\left(P-\varepsilon D D^{T}\right)^{-1} A+\frac{1}{\varepsilon} E^{T} E .
$$

\section{Delay-Dependent Robust Model Predictive Controller}

Let us consider the controller synthesis for the linear system with multiple uncertain delayed states. The aim of the current paper is to construct a delay-dependent state feedback controller which can be represented by a function:

$$
\begin{aligned}
u(k+i \mid k)= & K(k) x(k+i \mid k) \\
& +\sum_{\tau=1}^{l} K_{d \tau}(k) x\left(k+i-\widetilde{d}_{\tau} \mid k\right),
\end{aligned}
$$

where $K(k), K_{d 1}(k), \ldots, K_{d l}(k)$ are gain matrices to be determined by MPC strategy. Note that the estimated delay indices $\tilde{d}_{\tau}$ involved in the control law are different from the real delay $d_{\tau}$. Since the value of the state is estimated at every sampling instant $k$, we also assume that the more general case $\tilde{d}_{\tau} \leq d_{\tau} \leq \tilde{d}_{\tau}+\eta_{\tau}$ and $\tilde{d}_{1} \leq \tilde{d}_{1}+\eta_{1} \leq \cdots \leq \tilde{d}_{\tau} \leq \tilde{d}_{\tau}+\eta_{\tau} \leq$ $\cdots \leq \widetilde{d}_{l} \leq \widetilde{d}_{l}+\eta_{l}$, with $\eta_{\tau}$ being estimated errors and satisfying $\left|\eta_{\tau}\right| \leq \delta(\tau=1, \ldots, l, \delta \geq 0)$.

Now, for system (1), the following terminology used for controller (7) is listed as follows. 
(i) If $K_{d \tau}=0$ for all $\tau=1, \ldots, l$, the controller is referred to as a memoryless controller consisting of only the current state.

(ii) If $d_{\tau}=\widetilde{d}_{\tau}$ for all $k \geq 0$ and $\tau=1, \ldots, l$ (i.e., $\delta=0$ ), then the controller is referred to as a delay-dependent exact-memory controller.

(iii) If $\left|d_{\tau}-\tilde{d}_{\tau}\right|<\delta$ for all $\tau=1, \ldots, l$ and $\delta>0$, then the controller is referred to as a delay-dependent controller with estimated time-delayed indices.

In order to design such a controller (7), the following infinite horizon quadratic cost function is first defined:

$$
J(k)=\sum_{i=0}^{\infty}\|x(k+i \mid k)\|_{\mathrm{Q}_{1}}+\|u(k+i \mid k)\|_{R},
$$

where $Q_{1}, R$ are given positive definite symmetric matrices. Consider the following min-max optimization problem concluding the estimated time-delay indices, which minimizes the worst case infinite horizon quadratic objective function:

$$
\min _{u(k+i \mid k)=K(k) x(k+i \mid k)+\sum_{\tau=1}^{l} K_{d \tau}(k) x\left(k+i-\widetilde{d}_{\tau} \mid k\right)}\left[\max _{[A(k+i), B(k+i)], i>0} J(k)\right],
$$

where $x(k+i \mid k)$ and $u(k+i \mid k)$ are the predicted state and input, respectively, with $x(k \mid k)=x(k), x(k+i \mid k)=x(k+i)$ for $i \geq 0$.

Now, we propose a new MPC algorithm for a multiple uncertain state delayed system (1), in which the gain matrices $K(k)$ and $K_{d 1}(k), \ldots, K_{d l}(k)$ of the memory state feedback controller (7) are determined from the new sufficient condition for cost monotonicity. The sufficient condition is derived using the delayed state dependent quadratic function. Comparing with previous results [15], the proposed method allows an improvement in system performance in the sense of minimizing the upper bound of the infinite horizon cost $J(k)$, by taking the information of the multiple delayed states into full consideration. For the uncertain delayed indices, we consider the estimated errors and take them into account in an efficient way in the controller synthesis.

The goal of this paper is to find the stabilizing state feedback controller (7) for system (1) by the MPC strategy. And we need to determine the upper bound of the cost (8). Now, based on Lyapunov-Krasovskii function, we define the following delay-dependent quadratic function:

$$
\begin{aligned}
V(x(k))= & \|x(k)\|_{P}+\sum_{\tau=1}^{l} \sum_{j=1}^{\widetilde{d}_{\tau}}\|x(k-j \mid k)\|_{S_{\tau}} \\
& +\sum_{\tau=1}^{l} \sum_{j=\widetilde{d}_{\tau}+1}^{\tilde{d}_{\tau}+\eta_{\tau}}\|x(k-j \mid k)\|_{Z_{\tau}}
\end{aligned}
$$

where $P=P^{T}>0, S_{\tau}=S_{\tau}^{T}>0$, and $Z_{\tau}=Z_{\tau}^{T}>0(\tau=$ $1, \ldots, l)$ are positive definite symmetric matrices and satisfy the following inequality for given structured uncertainties $[\Delta A, \Delta B]$ :

$$
\begin{aligned}
\Delta V(i, k) & =V(x(k+i+1 \mid k))-V(x(k+i \mid k)) \\
& \leq-\|x(k+i \mid k)\|_{Q_{1}}-\|u(k+i \mid k)\|_{R} .
\end{aligned}
$$

Then, the cost monotonicity is guaranteed for the uncertain linear system (1). For the cost in (8) to be finite, it requires $V(x(\infty, k))=0$. Summing both sides of inequality (11) from $i=0$ to $i=\infty$, the worst value of the cost function (8) is bounded by

$$
\begin{aligned}
& \max _{[\Delta A, \Delta B]=H F(\cdot)\left[E_{1}, E_{2}\right]} J(k) \\
& \leq V(x(k, k))=\|x(k \mid k)\|_{P}+\sum_{\tau=1}^{l} \sum_{j=1}^{\widetilde{d}_{\tau}}\|x(k-j \mid k)\|_{S_{\tau}} \\
& \\
& \quad+\sum_{\tau=1}^{l} \sum_{j=\widetilde{d}_{\tau}+1}^{\widetilde{d}_{\tau}+\eta_{\tau}}\|x(k-j \mid k)\|_{Z_{\tau}} \cdot
\end{aligned}
$$

Therefore, the optimization is formulated as

$$
\begin{aligned}
\min _{P, S_{1}, \ldots, S_{l}, Z_{1}, \ldots, Z_{l}} & {\left[\|x(k \mid k)\|_{P}+\sum_{\tau=1}^{l} \sum_{j=1}^{\tilde{d}_{\tau}}\|x(k-j \mid k)\|_{S_{\tau}}\right.} \\
& \left.+\sum_{\tau=1}^{l} \sum_{j=\widetilde{d}_{\tau}+1}^{\tilde{d}_{\tau}+\eta_{\tau}}\|x(k-j \mid k)\|_{Z_{\tau}}\right]
\end{aligned}
$$

subject to (1), (7), (11) and (12).

In order to convert the minimization problem (13) into an LMI optimization problem that can be efficiently solved by convex optimization algorithm, here we define the upper bound of the object function in (12) as

$$
\begin{aligned}
& \|x(k \mid k)\|_{P}+\sum_{\tau=1}^{l} \sum_{j=1}^{\tilde{d}_{\tau}}\|x(k-j \mid k)\|_{S_{\tau}} \\
& +\sum_{\tau=1}^{l} \sum_{j=\tilde{d}_{\tau}+1}^{\tilde{d}_{\tau}+\eta_{\tau}}\|x(k-j \mid k)\|_{Z_{\tau}} \leq \gamma(k),
\end{aligned}
$$

where $\gamma(k)$ is the nonnegative upper bound of $V$ to be minimized. Based on (14), the original min-max problem (13) can be redefined as the following optimization problem:

$$
\min _{\gamma(k), P, S_{1}, \ldots, S_{l}, Z_{1}, \ldots, Z_{l}} \gamma(k)
$$




\section{Main Result}

This section is devoted to the stability analysis of linear uncertain systems with multiple uncertain time-delays, described by (1) and (2). The results are based on the extension of [7] to linear uncertain systems with uncertain time-delays. Then, we derive an LMI condition for the cost monotonicity (11) for the delay-dependent quadratic function (10) in the following theorem.

Theorem 3. Consider the nonlinear system (1). It is robustly asymptotically stable if there exist $Q=Q^{T}, W, W_{1}, \ldots, W_{l}$, $Y, Y_{1}, \ldots, Y_{l}, Z_{1}, \ldots, Z_{l}, M_{1}, \ldots, M_{l}$ and $\gamma(k)>0$ satisfying the following LMI:

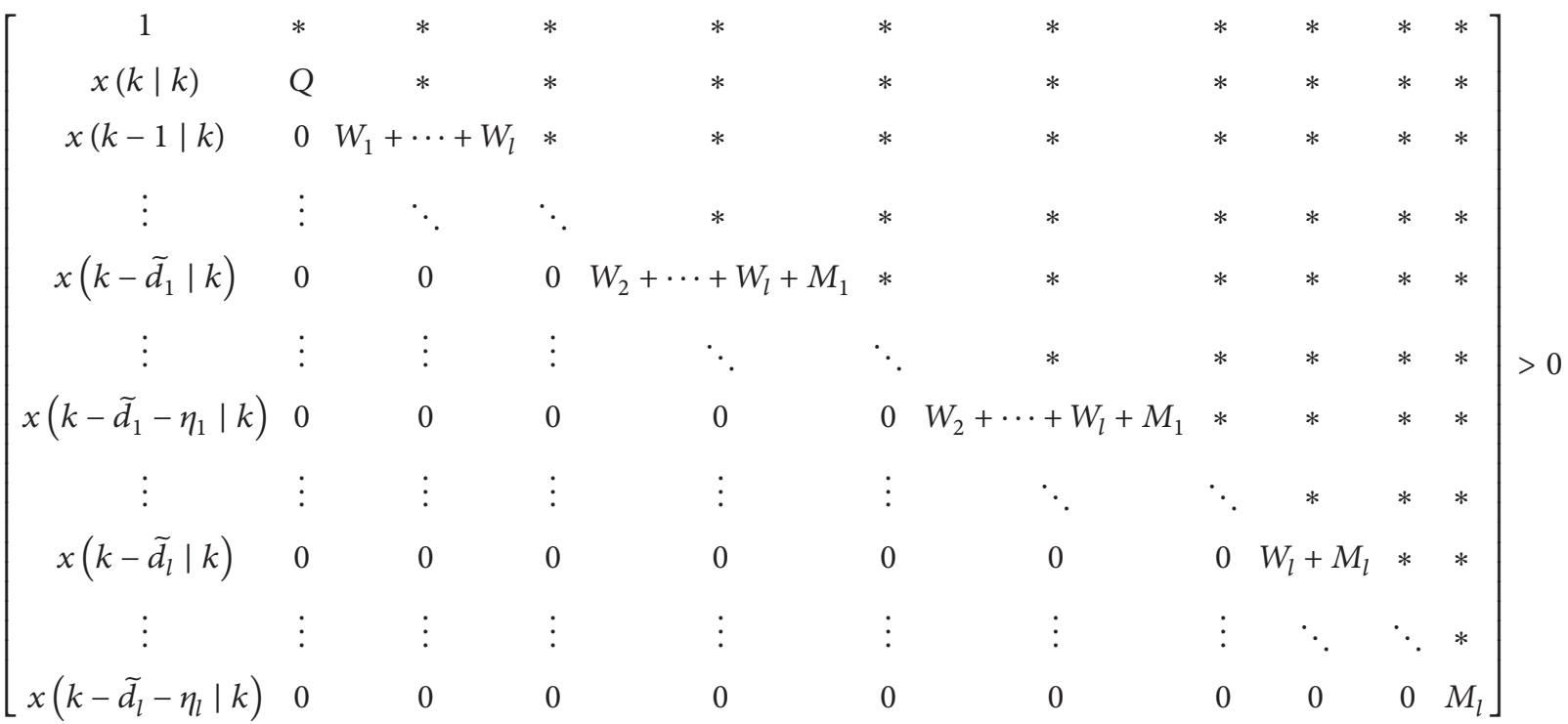

$$
\begin{aligned}
& {\left[\begin{array}{ccccc}
u_{\max }^{2} I & * & * & * & * \\
Y^{T} & Q & * & * & * \\
Y_{1}^{T} & 0 & W_{1} & * & * \\
\vdots & \vdots & \ddots & \ddots & * \\
Y_{l}^{T} & 0 & \cdots & 0 & W_{l}
\end{array}\right] \geq 0} \\
& {\left[\begin{array}{cccccccc}
\Phi_{1,1} & * & * & * & * & * & * & * \\
\Phi_{2,1} & \Phi_{2,2} & * & * & * & * & * & * \\
\Phi_{3,1} & 0 & \Phi_{3,3} & * & * & * & * & * \\
\Phi_{4,1} & 0 & 0 & \Phi_{4,4} & * & * & * & * \\
\vdots & \vdots & \vdots & \ddots & \ddots & * & * & * \\
\Phi_{l+3,1} & 0 & 0 & 0 & 0 & \Phi_{l+3, l+3} & * & * \\
\Phi_{l+4,1} & 0 & 0 & 0 & 0 & 0 & \Phi_{l+4, l+4} & * \\
\Phi_{l+5,1} & 0 & 0 & 0 & 0 & 0 & 0 & \Phi_{l+5, l+5}
\end{array}\right]>0} \\
& {\left[\begin{array}{cc}
Q & * \\
H^{T} & \Lambda^{-1}
\end{array}\right]>0,}
\end{aligned}
$$

where $\Phi_{1,1}=Q, \Phi_{2,1}=Q_{1}^{0.5} Q, \Phi_{3,1}=R^{0.5} Y, \Phi_{4,1}=\cdots=\quad \Phi_{3,3}=\gamma /(l+1), \Phi_{4,4}=W_{1}, \Phi_{l+3, l+3}=W_{l}, \Phi_{l+4, l+4}=\Lambda /(2 l+1)$, $\Phi_{l+3,1}=Q, \Phi_{l+4,1}=E_{1} Q+E_{2} Y, \Phi_{l+5,1}=A Q+B Y, \Phi_{2,2}=\gamma, \quad \Phi_{l+5, l+5}=\left(Q-\Lambda H H^{T}\right) /(2 l+1)$. 


$$
\begin{aligned}
& {\left[\begin{array}{ccccc}
W_{\tau} & * & * & * & * \\
R^{0.5} Y_{\tau} & \frac{1}{l+1} \gamma & * & * & * \\
E_{2} Y_{\tau} & 0 & \frac{1}{2 l+2} \Lambda_{\tau} & * & * \\
W_{\tau} & 0 & 0 & M_{\tau} & * \\
B Y_{\tau} & 0 & 0 & 0 & \frac{1}{2 l+2}\left(Q-\Lambda_{\tau} H H^{T}\right)
\end{array}\right]} \\
& >0 \quad(\tau=1, \ldots, l) \\
& {\left[\begin{array}{cc}
Q & * \\
H^{T} & \Lambda_{\tau}^{-1}
\end{array}\right]>0 \quad(\tau=1, \ldots, l)} \\
& {\left[\begin{array}{cc}
M_{\tau} & * \\
A_{d \tau}^{T} & \frac{1}{2 l+2} Q
\end{array}\right]>0 \quad(\tau=1, \ldots, l)}
\end{aligned}
$$

Proof. Firstly, we derive the LMI condition for inequality (14). Inequality (14) is rewritten as

$$
\begin{aligned}
& \|x(k \mid k)\|_{P} \\
& +\left[\sum_{j=1}^{\tilde{d}_{1}}\|x(k-j \mid k)\|_{S_{1}}+\cdots+\sum_{j=1}^{\tilde{d}_{l}}\|x(k-j \mid k)\|_{S_{l}}\right]
\end{aligned}
$$

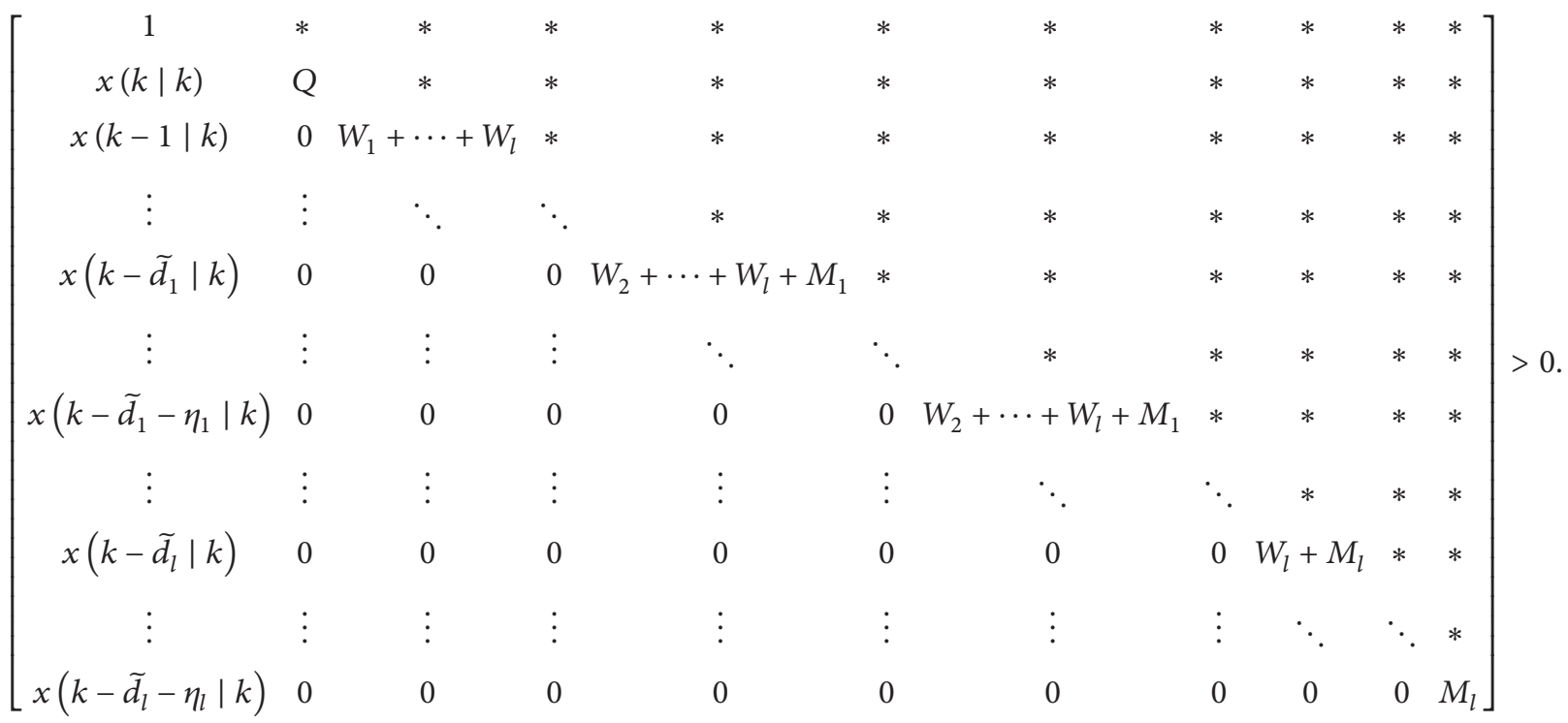

$$
\begin{aligned}
& +\left[\sum_{j=\tilde{d}_{1}+1}^{\widetilde{d}_{1}+\eta_{1}}\|x(k-j \mid k)\|_{Z_{1}}+\cdots+\sum_{j=\tilde{d}_{l}+1}^{\tilde{d}_{l}+\eta_{l}}\|x(k-j \mid k)\|_{Z_{l}}\right] \\
& \leq \gamma(k) .
\end{aligned}
$$

Multiplying both sides of inequality (18) by $\gamma^{-1}(k)$, we obtain that

$$
\begin{aligned}
& \|x(k \mid k)\|_{\gamma^{-1} P} \\
& +\left[\sum_{j=1}^{\tilde{d}_{1}}\|x(k-j \mid k)\|_{\gamma^{-1} S_{1}}+\cdots+\sum_{j=1}^{\tilde{d}_{l}}\|x(k-j \mid k)\|_{\gamma^{-1} S_{l}}\right] \\
& +\left[\sum_{j=\tilde{d}_{1}+1}^{\tilde{d}_{1}+\eta_{1}}\|x(k-j \mid k)\|_{\gamma^{-1} Z_{1}}\right. \\
& \left.+\cdots+\sum_{j=\tilde{d}_{l}+1}^{\tilde{d}_{l}+\eta_{l}}\|x(k-j \mid k)\|_{\gamma^{-1} Z_{l}}\right] \leq 1 .
\end{aligned}
$$

Choosing $P=\gamma Q^{-1}, S_{1}=\gamma W_{1}^{-1}, \ldots, S_{l}=\gamma W_{l}^{-1}, Z_{1}=$ $\gamma M_{1}^{-1}, \ldots, Z_{l}=\gamma M_{l}^{-1}$ and using Schur complement in [19], we have
The differential of the quadratic function $V(x(k+j))$ is found by substituting (10) into (11):

$$
\begin{aligned}
& \Delta V(i, k) \\
& =V(x(k+i+1 \mid k))-V(x(k+i \mid k)) \\
& =\|x(k+i+1 \mid k)\|_{P}-\|x(k+i \mid k)\|_{P}
\end{aligned}
$$

$$
\begin{aligned}
& +\|x(k+i \mid k)\|_{S_{1}}+\cdots+\|x(k+i \mid k)\|_{S_{l}} \\
& +\left[\left\|x\left(k+i-\tilde{d}_{1} \mid k\right)\right\|_{Z_{1}}-\left\|x\left(k+i-\widetilde{d}_{1} \mid k\right)\right\|_{S_{1}}\right] \\
& +\cdots+\left[\left\|x\left(k+i-\widetilde{d}_{l} \mid k\right)\right\|_{Z_{l}}-\left\|x\left(k+i-\tilde{d}_{l} \mid k\right)\right\|_{S_{l}}\right] \\
& -\left\|x\left(k+i-\tilde{d}_{1}-\eta_{1} \mid k\right)\right\|_{Z_{1}}
\end{aligned}
$$




$$
\begin{gathered}
-\cdots-\left\|x\left(k+i-\tilde{d}_{l}-\eta_{l} \mid k\right)\right\|_{Z_{l}} \\
\leq-\|x(k+i \mid k)\|_{Q_{1}}-\|u(k+i \mid k)\|_{R} .
\end{gathered}
$$

From system (1) and the delay-dependent state feedback controller (7), replacing the delay indices $d_{\tau}$ with $\widetilde{d}_{\tau}+\eta_{\tau}$, we get

$$
\begin{aligned}
& x(k+i+1 \mid k) \\
& =A(k+i) x(k+i \mid k)+\sum_{\tau=1}^{l} A_{d \tau} x\left(k+i-\tilde{d}_{\tau}-\eta_{\tau} \mid k\right) \\
& +B(k+i)[K(k) x(k+i \mid k) \\
& \left.\quad+\sum_{\tau=1}^{l} K_{d \tau}(k) x\left(k+i-\tilde{d}_{\tau} \mid k\right)\right] \\
& =A_{K} x(k+i \mid k) \\
& +\sum_{\tau=1}^{l}\left[A_{d \tau} x\left(k+i-\tilde{d}_{\tau}-\eta_{\tau} \mid k\right)\right. \\
& \left.+B(k+i) K_{d \tau}(k) x\left(k+i-\tilde{d}_{\tau} \mid k\right)\right]
\end{aligned}
$$

where $A_{K}=A(k+i)+B(k+i) K(k)$. Then, we obtain $\| x(k+i+1 \mid$ $k) \|_{P}$ described as the following inequality:

$$
\begin{aligned}
& \|x(k+i+1 \mid k)\|_{P} \\
& =\| A_{K} x(k+i \mid k) \\
& \quad+\sum_{\tau=1}^{l}\left[A_{d \tau} x\left(k+i-\tilde{d}_{\tau}-\eta_{\tau} \mid k\right)\right. \\
& \left.\quad+B(k+i) K_{d \tau}(k) x\left(k+i-\tilde{d}_{\tau} \mid k\right)\right] \|_{P}
\end{aligned}
$$

Thus, applying (23) into (21) yields

$$
\begin{aligned}
& \Delta V(i, k) \\
& =\left[A_{K} x(k+i \mid k)+\sum_{\tau=1}^{l} A_{d \tau} x\left(k+i-\widetilde{d}_{\tau}-\eta_{\tau} \mid k\right)\right.
\end{aligned}
$$

$$
\begin{aligned}
& \left.+B(k+i) K_{d \tau}(k) x\left(k+i-\tilde{d}_{\tau} \mid k\right)\right]^{T} \\
& +P\left[A_{K} x(k+i \mid k)+\sum_{\tau=1}^{l} A_{d \tau} x\left(k+i-\tilde{d}_{\tau}-\eta_{\tau} \mid k\right)\right. \\
& +\|x(k+i \mid k)\|_{P} \\
& +\|x(k+i \mid k)\|_{S_{1}}+\cdots+\|x(k+i \mid k)\|_{S_{l}} \\
& +\left[\left\|x\left(k+i-\tilde{d}_{1} \mid k\right)\right\|_{Z_{1}}-\left\|x\left(k+i-\tilde{d}_{1} \mid k\right)\right\|_{S_{1}}\right] \\
& +\cdots+\left[\left\|x\left(k+i-\tilde{d}_{l} \mid k\right)\right\|_{Z_{l}}-\left\|x\left(k+i-\tilde{d}_{l} \mid k\right)\right\|_{S_{l}}\right] \\
& +\left\|x\left(k+i-\tilde{d}_{1}-\eta_{1} \mid k\right)\right\|_{Z_{1}} \\
& +\cdots-\left\|x\left(k+i-\tilde{d}_{l}-\eta_{l} \mid k\right)\right\|_{Z_{l}} \\
& \leq-\|x(k+i \mid k)\|_{Q_{1}}-\|u(k+i \mid k)\|_{R} \cdot
\end{aligned}
$$

Then, inequality (24) in matrix form is rewritten as

$\Delta V(i, k)$

$$
\begin{aligned}
& =\mathbf{X}^{T}\left[\begin{array}{ccccccc}
\Xi_{1,1} & * & * & * & * & * & * \\
\Xi_{2,1} & \Xi_{2,2} & * & * & * & * & * \\
\Xi_{3,1} & \Xi_{2,3} & \Xi_{3,3} & * & * & * & * \\
\vdots & \vdots & \vdots & \ddots & * & * & * \\
\vdots & \vdots & \vdots & \vdots & \ddots & * & * \\
\Xi_{2 l, 1} & \Xi_{2 l, 2} & \Xi_{2 l, 3} & \cdots & \cdots & \Xi_{2 l, 2 l} & * \\
\Xi_{2 l+1,1} & \Xi_{2 l+1,2} & \Xi_{2 l+1,3} & \cdots & \cdots & \Xi_{2 l, 2 l+1} & \Xi_{2 l+1,2 l+1}
\end{array}\right] \mathbf{X} \\
& \leq-\|x(k+i \mid k)\|_{Q_{1}}-\|u(k+i \mid k)\|_{R},
\end{aligned}
$$

where $\mathbf{X}^{T}=\left[x(k+i \mid k) \quad x\left(k+i-\tilde{d}_{1} \mid k\right) \quad x\left(k+i-\tilde{d}_{1}-\eta_{1} \mid\right.\right.$ k) $\left.\quad \cdots \quad \cdots \quad x\left(k+i-\tilde{d}_{l} \mid k\right) \quad x\left(k+i-\tilde{d}_{l}-\eta_{l} \mid k\right)\right]^{T}$, $\Xi_{1,1}=A_{K}^{T} P A_{K}-P+S_{1}+\cdots+S_{l}, \Xi_{2,1}=A_{K}^{T} P B(k+i) K_{d 1}$, $\Xi_{3,1}=A_{K}^{T} P A_{d 1}, \Xi_{2 l, 1}=A_{K}^{T} P B(k+i) K_{d l}, \Xi_{2 l+1,1}=A_{K}^{T} P A_{d l}$, $\Xi_{2,2}=\left[B(k+i) K_{d 1}\right]^{T} P\left[B(k+i) K_{d 1}\right]+Z_{1}-S_{1}, \Xi_{3,3}=A_{d 1}^{T} P A_{d 1}-$ $Z_{1}, \Xi_{2,3}=\left[B(k+i) K_{d 1}\right]^{T} P A_{d 1}, \Xi_{2 l, 2}=\left[B(k+i) K_{d 1}\right]^{T} P B(k+$ i) $K_{d l}, \Xi_{2 l+1,2}=\left[B(k+i) K_{d 1}\right]^{T} P A_{d l}, \Xi_{2 l, 3}=A_{d 1}^{T} P B(k+i) K_{d l}$, $\Xi_{2 l+1,3}=A_{d 1}^{T} P A_{d l}, \Xi_{2 l, 2 l}=\left[B(k+i) K_{d l}\right]^{T} P\left[B(k+i) K_{d l}\right]+Z_{l}-S_{l}$, $\Xi_{2 l, 2 l+1}=\left[B(k+i) K_{d l}\right]^{T} P A_{d l}, \Xi_{2 l+1,2 l+1}=A_{d l}^{T} P A_{d l}-Z_{l}$. 
Then, from (7) we can further obtain that

$$
\begin{aligned}
\|u(k+i \mid k)\|_{R} \\
=\left[K(k) x(k+i \mid k)+\sum_{\tau=1}^{l} K_{d \tau}(k) x\left(k+i-d_{\tau} \mid k\right)\right]^{T} \\
\cdot R\left[K(k) x(k+i \mid k)+\sum_{\tau=1}^{l} K_{d \tau}(k) x\left(k+i-d_{\tau} \mid k\right)\right] \\
=\left[\begin{array}{c}
x(k+i \mid k) \\
x\left(k+i-\tilde{d}_{1} \mid k\right) \\
x\left(k+i-\tilde{d}_{1}-\eta_{1} \mid k\right) \\
\vdots \\
x\left(k+i-\tilde{d}_{l} \mid k\right) \\
x\left(k+i-\tilde{d}_{l}-\eta_{l} \mid k\right)
\end{array}\right]^{T}
\end{aligned}
$$$$
\begin{aligned}
& {\left[\begin{array}{ccccccc}
K^{T} R K & * & * & * & * & * & * \\
K^{T} R K_{d 1} & K_{d 1}^{T} R K_{d 1} & * & * & * & * & * \\
0 & 0 & 0 & * & * & * & * \\
\vdots & \vdots & \vdots & \ddots & * & * & * \\
\vdots & \vdots & \vdots & \cdots & \ddots & * & * \\
K^{T} R K_{d l} & K_{d 1}^{T} R K_{d l} & 0 & \cdots & \cdots & K_{d l}^{T} R K_{d l} & * \\
0 & 0 & 0 & \cdots & \cdots & 0 & 0
\end{array}\right]} \\
& \cdot\left[\begin{array}{c}
x(k+i \mid k) \\
x\left(k+i-\tilde{d}_{1} \mid k\right) \\
x\left(k+i-\tilde{d}_{1}-\eta_{1} \mid k\right) \\
\vdots \\
x\left(k+i-\tilde{d}_{l} \mid k\right) \\
x\left(k+i-\tilde{d}_{l}-\eta_{l} \mid k\right)
\end{array}\right]
\end{aligned}
$$

Therefore, equality (26) can be rewritten as

$$
\|u(k+i \mid k)\|_{R}=\mathbf{X}^{T}\left[\begin{array}{ccccccc}
\Psi_{1,1} & * & * & * & * & * & * \\
\Psi_{2,1} & \Psi_{2,2} & * & * & * & * & * \\
\Psi_{3,1} & \Psi_{3,2} & \Psi_{3,3} & * & * & * & * \\
\vdots & \vdots & \vdots & \ddots & * & * & * \\
\vdots & \vdots & \vdots & \cdots & \ddots & * & * \\
\Psi_{2 l, 1} & \Psi_{2 l, 2} & \Psi_{2 l, 3} & \cdots & \cdots & \Psi_{2 l, 2 l} & * \\
\Psi_{2 l+1,1} & \Psi_{2 l+1,2} & \Psi_{2 l+1,3} & \cdots & \cdots & \Psi_{2 l, 2 l+1} & \Psi_{2 l+1,2 l+1}
\end{array}\right] \mathbf{X}
$$

where $\Psi_{1,1}=K^{T} R K, \Psi_{2,1}=K^{T} R K_{d 1}, \Psi_{3,1}=0, \Psi_{2 l, 1}=$ $K^{T} R K_{d l}, \Psi_{2 l+1,1}=0, \Psi_{2,2}=K_{d 1}^{T} R K_{d 1}, \Psi_{3,2}=0, \Psi_{2 l, 2}=$
$K_{d 1}^{T} R K_{d l}, \Psi_{2 l+1,2}=0, \Psi_{3,3}=0, \Psi_{2 l, 3}=0, \Psi_{2 l+1,3}=0$, $\Psi_{2 l, 2 l}=K_{d l}^{T} R K_{d l}, \Psi_{2 l+1,2 l}=0, \Psi_{2 l+1,2 l+1}=0$.

Substituting (23) into (21) and from (27), the following holds:

$\Delta V(i, k)+\|x(k+i \mid k)\|_{Q_{1}}+\|u(k+i \mid k)\|_{R}$

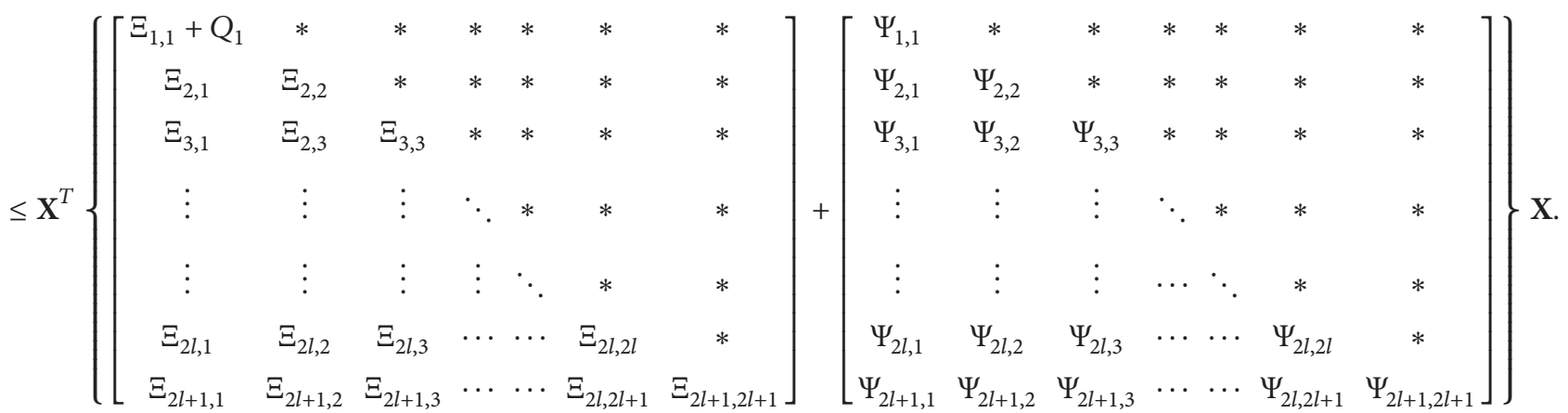


It is easy to see that inequality (28) is equivalent to

$\Delta V(i, k)+\|x(k+i \mid k)\|_{Q_{1}}+\|u(k+i \mid k)\|_{R}$

$\leq \mathbf{X}^{T}\left\{\left[\begin{array}{ccccccc}M_{1,1} & * & * & * & * & * & * \\ M_{2,1} & M_{2,2} & * & * & * & * & * \\ M_{3,1} & M_{3,2} & M_{3,3} & * & * & * & * \\ \vdots & \vdots & \vdots & \ddots & * & * & * \\ \vdots & \vdots & \vdots & \ddots & \ddots & * & * \\ M_{2 l, 1} & M_{2 l, 2} & M_{2 l, 3} & \cdots & \cdots & M_{2 l, 2 l} & * \\ M_{2 l+1,1} & M_{2 l+1,2} & M_{2 l+1,3} & \cdots & \cdots & M_{2 l, 2 l+1} & M_{2 l+1,2 l+1}\end{array}\right]+\left[\begin{array}{ccccccc}K^{T} R K & * & * & * & * & * & * \\ K^{T} R K_{d 1} & K_{d 1}^{T} R K_{d 1} & * & * & * & * & * \\ 0 & 0 & 0 & * & * & * & * \\ \vdots & \vdots & \vdots & \ddots & * & * & * \\ \vdots & \vdots & \vdots & \cdots & \ddots & * & * \\ K^{T} R K_{d l} & K_{d 1}^{T} R K_{d l} & 0 & \cdots & \cdots & K_{d l}^{T} R K_{d l} & * \\ 0 & 0 & 0 & \cdots & \cdots & 0 & 0\end{array}\right]\right.$ $\left.+\left[\begin{array}{ccccccc}Q_{1}-P+S_{1}+\cdots+S_{l} & * & * & * & * & * & * \\ 0 & Z_{1}-S_{1} & * & * & * & * & * \\ 0 & 0 & -Z_{1} & * & * & * & * \\ \vdots & \vdots & \vdots & \ddots & * & * & * \\ \vdots & \vdots & \vdots & \ddots & \ddots & * & * \\ 0 & 0 & 0 & \cdots & \cdots & Z_{l}-S_{l} & * \\ 0 & 0 & 0 & \cdots & \cdots & 0 & -Z_{l}\end{array}\right]\right\} \mathbf{X}$

$=\mathbf{X}^{T}\left(\bar{M}^{\prime}+M^{\prime}\right) \mathbf{X}$

where $M_{1,1}=A_{K}^{T} P A_{K}+K^{T} R K, M_{2,1}=A_{K}^{T} P B(k+i) K_{d 1}+$ $K^{T} R K_{d 1}, M_{3,1}=A_{K}^{T} P A_{d 1}, M_{2 l, 1}=A_{K}^{T} P B(k+i) K_{d l}+K^{T} R K_{d l}$, $M_{2 l+1,1}=A_{K}^{T} P A_{d l}, M_{2,2}=\left[B(k+i) K_{d 1}\right]^{T} P\left[B(k+i) K_{d 1}\right]+$ $K_{d 1}^{T} R K_{d 1}, M_{3,3}=A_{d 1}^{T} P A_{d 1}, M_{2,3}=\left[B(k+i) K_{d 1}\right]^{T} P A_{d 1}$, $M_{2 l, 2}=\left[B(k+i) K_{d 1}\right]^{T} P B(k+i) K_{d l}, M_{2 l+1,2}=[B(k+$ i) $\left.K_{d 1}\right]^{T} P A_{d l}+K_{d 1}^{T} R K_{d l}, M_{2 l, 3}=A_{d 1}^{T} P B(k+i) K_{d l}, M_{2 l+1,3}=$ $A_{d 1}^{T} P A_{d l}, M_{2 l, 2 l}=\left[B(k+i) K_{d l}\right]^{T} P\left[B(k+i) K_{d l}\right]+K_{d l}^{T} R K_{d l}$, $M_{2 l, 2 l+1}=\left[B(k+i) K_{d l}\right]^{T} P A_{d l}, M_{2 l+1,2 l+1}=A_{d l}^{T} P A_{d l}$.

From Lemma 1, we have the following inequalities:

$$
\begin{aligned}
& x^{T}(k+i \mid k) M_{1,1} x(k+i \mid k)\|K x(k+i \mid k)\|_{R}+\left\|A_{K} x(k+i \mid k)\right\|_{P} \\
& x^{T}(k+i \mid k) M_{2 j, 1} x\left(k+i-\tilde{d}_{j} \mid k\right) \\
& \leq \frac{1}{2}\left[\left\|A_{K} x(k+i \mid k)\right\|_{P}+\left\|B(k+i) K_{d j} x\left(k+i-\tilde{d}_{j} \mid k\right)\right\|_{P}\right. \\
&\left.\quad+\|K x(k+i \mid k)\|_{R}+\left\|K_{d j} x\left(k+i-\tilde{d}_{j} \mid k\right)\right\|_{R}\right]
\end{aligned}
$$$$
(j=1, \ldots, l)
$$

$x^{T}(k+i \mid k) M_{2 j+1,1} x\left(k+i-\tilde{d}_{j}-\eta_{j} \mid k\right)$

$$
\begin{aligned}
& \leq \frac{1}{2}\left[\left\|A_{K} x(k+i \mid k)\right\|_{P}+\left\|A_{d j}(k) x\left(k+i-\tilde{d}_{j}-\eta_{j} \mid k\right)\right\|_{P}\right] \\
& (j=1, \ldots, l) \\
& x\left(k+i-\widetilde{d}_{j} \mid k\right) M_{2 j, 2 \varsigma} x\left(k+i-\widetilde{d}_{\varsigma} \mid k\right) \\
& \leq \frac{1}{2}\left[\left\|B(k+i) K_{d j} x\left(k+i-\tilde{d}_{j} \mid k\right)\right\|_{P}\right. \\
& +\left\|B(k+i) K_{d \varsigma} x\left(k+i-\tilde{d}_{\varsigma} \mid k\right)\right\|_{P} \\
& +\left\|K_{d j} x\left(k+i-\widetilde{d}_{j} \mid k\right)\right\|_{R} \\
& \left.+\left\|K_{d \varsigma} x\left(k+i-\tilde{d}_{\varsigma} \mid k\right)\right\|_{R}\right] \\
& (j=1, \ldots, l, \varsigma=j, \ldots, l) \\
& x^{T}\left(k+i-\tilde{d}_{j} \mid k\right) M_{2 j, 2 \varsigma+1} x\left(k+i-\tilde{d}_{\varsigma}-\eta_{\varsigma} \mid k\right) \\
& \leq \frac{1}{2}\left\{\left\|B(k+i) K_{d j} x\left(k+i-\tilde{d}_{j} \mid k\right)\right\|_{P}\right. \\
& \left.+\left\|A_{d \varsigma} x\left(k+i-\widetilde{d}_{\varsigma}-\eta_{\varsigma} \mid k\right)\right\|_{P}\right\}
\end{aligned}
$$




$$
\begin{gathered}
(j=1, \ldots, l, \varsigma=j, \ldots, l) \\
x^{T}\left(k+i-\widetilde{d}_{j}-\eta_{j} \mid k\right) M_{2 j+1,2 \varsigma} x\left(k+i-\widetilde{d}_{\varsigma} \mid k\right) \\
\leq \frac{1}{2}\left\{\left\|A_{d j}(k) x\left(k+i-\widetilde{d}_{j}-\eta_{j} \mid k\right)\right\|_{P}\right. \\
\left.+\left\|B(k+i) K_{d \varsigma} x\left(k+i-\widetilde{d}_{\varsigma} \mid k\right)\right\|_{P}\right\} \\
(j=1, \ldots, l, \varsigma=j, \ldots, l) \\
x^{T}\left(k+i-\tilde{d}_{j}-\eta_{j} \mid k\right) M_{2 j+1,2 \varsigma+1} x\left(k+i-\tilde{d}_{\varsigma}-\eta_{\varsigma} \mid k\right) \\
\leq \frac{1}{2}\left\{\left\|A_{d j} x\left(k+i-\tilde{d}_{j}-\eta_{j} \mid k\right)\right\|_{P}\right. \\
\left.+\left\|A_{d \varsigma} x\left(k+i-\tilde{d}_{\varsigma}-\eta_{\varsigma} \mid k\right)\right\|_{P}\right\} \\
(j=1, \ldots, l, \varsigma=j, \ldots, l) .
\end{gathered}
$$

Hence, from inequalities (30), we obtain

$$
\begin{aligned}
& x^{T}(k+i \mid k) M_{1,1} x(k+i \mid k) \\
& +\sum_{j=1}^{l} x^{T}(k+i \mid k) M_{2 j, 1} x\left(k+i-\tilde{d}_{j} \mid k\right) \\
& +x^{T}(k+i \mid k) M_{2 j+1,1} x\left(k+i-\tilde{d}_{j}-\eta_{j} \mid k\right) \\
& \leq\|K x(k+i \mid k)\|_{R}+\left\|A_{K} x(k+i \mid k)\right\|_{P} \\
& +\frac{1}{2} \sum_{j=1}^{l}\left[2\left\|A_{K} x(k+i \mid k)\right\|_{P}+\|K x(k+i \mid k)\|_{R}\right. \\
& +\left\|B(k+i) K_{d j} x\left(k+i-\tilde{d}_{j} \mid k\right)\right\|_{P} \\
& +\left\|K_{d j} x\left(k+i-\tilde{d}_{j} \mid k\right)\right\|_{R} \\
& \left.+\left\|A_{d j} x\left(k+i-\tilde{d}_{j}-\eta_{j} \mid k\right)\right\|_{P}\right] \\
& \sum_{j=1}^{l} \sum_{\varsigma=j}^{l}\left[x\left(k+i-\widetilde{d}_{j} \mid k\right) M_{2 j, 2 \varsigma} x\left(k+i-\widetilde{d}_{\zeta} \mid k\right)\right. \\
& +x^{T}\left(k+i-\tilde{d}_{j} \mid k\right) \\
& \cdot M_{2 j, 2 \varsigma+1} x\left(k+i-\tilde{d}_{\varsigma}-\eta_{\varsigma} \mid k\right) \\
& +x^{T}\left(k+i-\widetilde{d}_{j}-\eta_{j} \mid k\right) \\
& \cdot M_{2 j+1,2 \varsigma} x\left(k+i-\widetilde{d}_{\varsigma} \mid k\right) \\
& +x^{T}\left(k+i-\tilde{d}_{j} \mid k\right) \\
& \text { - } \left.M_{2 j+1,2 \varsigma+1} x\left(k+i-\widetilde{d}_{\varsigma}-\eta_{\varsigma} \mid k\right)\right]
\end{aligned}
$$

$$
\begin{aligned}
\leq \frac{1}{2} \sum_{j=1}^{l} \sum_{\zeta=j}^{l}[\| & B(k+i) K_{d j} x\left(k+i-\tilde{d}_{j} \mid k\right) \|_{P} \\
& +\left\|B(k+i) K_{d \varsigma} x\left(k+i-\tilde{d}_{\varsigma} \mid k\right)\right\|_{P} \\
& +\left\|K_{d j} x\left(k+i-\tilde{d}_{j} \mid k\right)\right\|_{R} \\
& +\left\|K_{d \varsigma} x\left(k+i-\tilde{d}_{\varsigma} \mid k\right)\right\|_{R} \\
& +\left\|B(k+i) K_{d j} x\left(k+i-\tilde{d}_{j} \mid k\right)\right\|_{P} \\
& +\left\|A_{d \varsigma} x\left(k+i-\tilde{d}_{\varsigma}-\eta_{\varsigma} \mid k\right)\right\|_{P} \\
& +\left\|A_{d j} x\left(k+i-\tilde{d}_{j}-\eta_{j} \mid k\right)\right\|_{P} \\
& +\left\|B(k+i) K_{d \zeta} x\left(k+i-\tilde{d}_{\varsigma} \mid k\right)\right\|_{P} \\
& +\left\|A_{d j} x\left(k+i-\tilde{d}_{j}-\eta_{j} \mid k\right)\right\|_{P} \\
& \left.+\left\|A_{d \varsigma} x\left(k+i-\tilde{d}_{\varsigma}-\eta_{\varsigma} \mid k\right)\right\|_{P}\right]
\end{aligned}
$$$$
=\frac{1}{2} \sum_{j=1}^{l} \sum_{\zeta=j}^{l}\left[2\left\|B(k+i) K_{d j} x\left(k+i-\widetilde{d}_{j} \mid k\right)\right\|_{P}\right.
$$$$
+2\left\|B(k+i) K_{d \varsigma} x\left(k+i-\widetilde{d}_{\varsigma} \mid k\right)\right\|_{P}
$$$$
+\left\|K_{d j} x\left(k+i-\tilde{d}_{j} \mid k\right)\right\|_{R}
$$$$
+\left\|K_{d \varsigma} x\left(k+i-\widetilde{d}_{\varsigma} \mid k\right)\right\|_{R}
$$$$
+2\left\|A_{d j} x\left(k+i-\widetilde{d}_{j}-\eta_{j} \mid k\right)\right\|_{P}
$$$$
\left.+2\left\|A_{d \zeta} x\left(k+i-\widetilde{d}_{\varsigma}-\eta_{\varsigma} \mid k\right)\right\|_{P}\right]
$$$$
=\frac{l+1}{2} \sum_{j=1}^{l}\left[2\left\|B(k+i) K_{d j} x\left(k+i-\tilde{d}_{j} \mid k\right)\right\|_{P}\right.
$$$$
+\left\|K_{d j} x\left(k+i-\tilde{d}_{j} \mid k\right)\right\|_{R}
$$$$
\left.+2\left\|A_{d j} x\left(k+i-\widetilde{d}_{j}-\eta_{j} \mid k\right)\right\|_{P}\right] \text {. }
$$

Therefore, from (31), we can obtain that the following inequality holds true:

$$
\begin{aligned}
& \mathbf{X}^{T} \bar{M}^{\prime} \mathbf{X} \\
& \leq(2 l+1)\left\|A_{K} x(k+i \mid k)\right\|_{P}+(l+1)\|K x(k+i \mid k)\|_{R} \\
& \quad+\sum_{j=1}^{l}\left[\left\|B(k+i) K_{d j} x\left(k+i-\widetilde{d}_{j} \mid k\right)\right\|_{P}\right. \\
& \quad+\left\|K_{d j} x\left(k+i-\widetilde{d}_{j} \mid k\right)\right\|_{R}
\end{aligned}
$$




$$
\begin{gathered}
\left.+\left\|A_{d j} x\left(k+i-\tilde{d}_{j}-\eta_{j} \mid k\right)\right\|_{P}\right] \\
+\sum_{j=1}^{l}(2 l+1)\left\|B(k+i) K_{d j} x\left(k+i-\tilde{d}_{j} \mid k\right)\right\|_{P} \\
+l\left\|K_{d j} x\left(k+i-\widetilde{d}_{j} \mid k\right)\right\|_{R} \\
+(2 l+1)\left\|A_{d j} x\left(k+i-\widetilde{d}_{j}-\eta_{j} \mid k\right)\right\|_{P} \\
=(2 l+1)\left\|A_{K} x(k+i \mid k)\right\|_{P}+(l+1)\|K x(k+i \mid k)\|_{R} \\
+(l+1) \sum_{j=1}^{l}\left[2\left\|B(k+i) K_{d j} x\left(k+i-\tilde{d}_{j} \mid k\right)\right\|_{P}\right. \\
+\left\|K_{d j} x\left(k+i-\tilde{d}_{j} \mid k\right)\right\|_{R} \\
\left.+2\left\|A_{d j} x\left(k+i-\tilde{d}_{j}-\eta_{j} \mid k\right)\right\|_{P}\right] .
\end{gathered}
$$

Then, inequality (32) can be rewritten as the following matrix form:

$$
\begin{aligned}
& \mathbf{X}^{T} \bar{M}^{\prime} \mathbf{X} \\
& \leq \mathbf{X}^{T}\left[\begin{array}{ccccccc}
\Pi_{1,1} & * & * & * & * & * & * \\
0 & \Pi_{2,2} & * & * & * & * & * \\
0 & 0 & \Pi_{3,3} & * & * & * & * \\
0 & 0 & 0 & \ddots & * & * & * \\
0 & 0 & 0 & \cdots & \ddots & * & * \\
0 & 0 & 0 & \cdots & \cdots & \Pi_{2 l, 2 l} & * \\
0 & 0 & 0 & \cdots & \cdots & 0 & \Pi_{2 l+1,2 l+1}
\end{array}\right] \mathbf{X},
\end{aligned}
$$

where $\Pi_{1,1}=(2 l+1) A_{K}^{T} P A_{K}+(l+1) K^{T} R K, \Pi_{2,2}=(2 l+$ 2) $\left(B(k+i) K_{d 1}\right)^{T} P\left(B(k+i) K_{d 1}\right)+(l+1) K_{d 1}^{T} R K_{d 1}, \Pi_{3,3}=(2 l+$ 2) $A_{d 1}^{T} P A_{d 1}, \Pi_{2 l, 2 l}=(2 l+2)\left(B(k+i) K_{d l}\right)^{T} P\left(B(k+i) K_{d l}\right)+(l+$ 1) $K_{d l}^{T} R K_{d l}, \Pi_{2 l+1,2 l+1}=(2 l+2) A_{d l}^{T} P A_{d l}$.

From inequality (33), inequality (29) can be derived as the following form:

$\Delta V(x(k+i \mid k))$

$$
\left.\leq \mathbf{X}^{T}\left[\begin{array}{ccccccc}
\Pi_{1,1} & * & * & * & * & * & * \\
0 & \Pi_{2,2} & * & * & * & * & * \\
0 & 0 & \Pi_{3,3} & * & * & * & * \\
\vdots & \vdots & \vdots & \ddots & * & * & * \\
\vdots & \vdots & \vdots & \ddots & \ddots & * & * \\
0 & 0 & 0 & \cdots & \cdots & \Pi_{2 l, 2 l} & * \\
0 & 0 & 0 & \cdots & \cdots & 0 & \Pi_{2 l+1,2 l+1}
\end{array}\right]+\left[\begin{array}{rrrrrrr}
Q_{1}-P+S_{1}+\cdots+S_{l} & * & * & * & * & * & * \\
0 & Z_{1}-S_{1} & * & * & * & * & * \\
0 & -Z_{1} & * & * & * & * \\
0 & \vdots & \ddots & * & * & * \\
\vdots & & \vdots & & & & \\
\vdots & & \vdots & \ddots & \ddots & * & * \\
0 & 0 & 0 & \cdots & \cdots & Z_{l}-S_{l} & * \\
0 & & 0 & 0 & \cdots & 0 & -Z_{l}
\end{array}\right]\right] \mathbf{X} .
$$

Thus, inequality (34) is satisfied if

$$
\begin{gathered}
(2 l+1) A_{K}^{T} P A_{K}+(l+1) K^{T} R K+Q_{1}-P+S_{1}+\cdots+S_{l} \leq 0 \\
(2 l+2)\left(B(k+i) K_{d 1}\right)^{T} P\left(B(k+i) K_{d 1}\right) \\
+(l+1) K_{d 1}^{T} R K_{d 1}+Z_{1}-S_{1} \leq 0 \\
(2 l+2) A_{d 1}^{T} P A_{d 1}-Z_{1} \leq 0 \\
\vdots \\
(2 l+2)\left(B(k+i) K_{d l}\right)^{T} P\left(B(k+i) K_{d l}\right) \\
+(l+1) K_{d l}^{T} R K_{d l}+Z_{l}-S_{l} \leq 0 \\
(2 l+2) A_{d l}^{T} P A_{d l}-Z_{l} \leq 0
\end{gathered}
$$

holds. Substituting $A_{K}=A+\Delta A+(B+\Delta B) K(\tau=1, \ldots, l)$ and (4) into (33), by Lemma 2 , then we have

$$
\begin{aligned}
(2 l+1) & A_{K}^{T} P A_{K}+(l+1) K^{T} R K+Q_{1}-P+S_{1}+\cdots+S_{l} \\
= & (2 l+1)\left[A+B K+H F(k)\left(E_{1}+E_{2} K\right)\right]^{T} \\
& \cdot P\left[A+B K+H F(k)\left(E_{1}+E_{2} K\right)\right] \\
& +(l+1) K^{T} R K+Q_{1}-P+S_{1}+\cdots+S_{l} \\
\leq & (2 l+1)\left[(A+B K)^{T}\left(P^{-1}-\varepsilon H H^{T}\right)^{-1}\right. \\
& \left.\cdot(A+B K)+\varepsilon^{-1}\left(E_{1}+E_{2} K\right)^{T}\left(E_{1}+E_{2} K\right)\right] \\
& +(l+1) K^{T} R K+Q_{1}-P+S_{1}+\cdots+S_{l} \leq 0
\end{aligned}
$$




$$
\begin{gathered}
(2 l+2)\left(B(k+i) K_{d \tau}\right)^{T} P\left(B(k+i) K_{d \tau}\right) \\
+(l+1) K_{d \tau}^{T} R K_{d \tau}+Z_{\tau}-S_{\tau} \leq 0 \\
(2 l+2) A_{d \tau}^{T} P A_{d \tau}-Z_{\tau} \leq 0 \\
(\tau=1, \ldots, l) .
\end{gathered}
$$

Multiplying both sides of inequality (36) by $Q^{T}$ and $\gamma^{-1} Q$, (36) equals the following inequality:

$$
\begin{aligned}
&(2 l+1) {\left[(A Q+B K Q)^{T}\left(\gamma P^{-1}-\gamma \varepsilon H^{T} H\right)^{-1}(A Q+B K Q)\right] } \\
&+(2 l+1)\left[\frac{1}{\gamma \varepsilon}\left(E_{1} Q+E_{2} K Q\right)^{T}\left(E_{1} Q+E_{2} K Q\right)\right] \\
&+ \frac{(l+1)}{\gamma}(K Q)^{T} R(K Q)+Q^{T} \frac{Q_{1}}{\gamma} Q-Q^{T} \frac{P}{\gamma} Q+Q^{T} \frac{S_{1}}{\gamma} Q \\
&+\cdots+Q^{T} \frac{S_{l}}{\gamma} Q \leq 0 .
\end{aligned}
$$

Now, choose $P^{-1}=\gamma^{-1} Q, S_{1}^{-1}=\gamma^{-1} W_{1}, \ldots, S_{l}^{-1}=\gamma^{-1} W_{l}, \Lambda=$ $\gamma \varepsilon, K Q=Y$; then by using Schur complement [19], inequality (36) can be derived as the following inequalities:

$$
\left[\begin{array}{cccccccc}
\Phi_{1,1} & * & * & * & * & * & * & * \\
\Phi_{2,1} & \Phi_{2,2} & * & * & * & * & * & * \\
\Phi_{3,1} & 0 & \Phi_{3,3} & * & * & * & * & * \\
\Phi_{4,1} & 0 & 0 & \Phi_{4,4} & * & * & * & * \\
\vdots & \vdots & \vdots & \vdots & \ddots & * & * & * \\
\Phi_{l+3,1} & 0 & 0 & 0 & 0 & \Phi_{l+3, l+3} & * & * \\
\Phi_{l+4,1} & 0 & 0 & 0 & 0 & 0 & \Phi_{l+4, l+4} & * \\
\Phi_{l+5,1} & 0 & 0 & 0 & 0 & 0 & 0 & \Phi_{l+5, l+5}
\end{array}\right]
$$$$
>0
$$

$$
\left[\begin{array}{cc}
Q & * \\
H^{T} & \Lambda^{-1}
\end{array}\right]>0,
$$

where $\Phi_{1,1}=Q, \Phi_{2,1}=Q_{1}^{0.5} Q, \Phi_{3,1}=R^{0.5} Y, \Phi_{4,1}=\cdots=$ $\Phi_{l+3,1}=Q, \Phi_{l+4,1}=E_{1} Q+E_{2} Y, \Phi_{l+5,1}=A Q+B Y, \Phi_{2,2}=\gamma$, $\Phi_{3,3}=\gamma /(l+1), \Phi_{4,4}=W_{1}, \Phi_{l+3, l+3}=W_{l}, \Phi_{l+4, l+4}=\Lambda /(2 l+1)$, $\Phi_{l+5, l+5}=\left(Q-\Lambda H H^{T}\right) /(2 l+1)$.

From (37) and by Lemma 2, we can obtain

$$
\begin{aligned}
(2 l+ & 2)\left(B K_{d \tau}\right)^{T}\left(P^{-1}-\varepsilon_{\tau} H H^{T}\right)^{-1}\left(B K_{d \tau}\right) \\
& +(2 l+2) \varepsilon_{\tau}^{-1}\left(E_{2} K_{d \tau}\right)^{T}\left(E_{2} K_{d \tau}\right)+(l+1) K_{d \tau}^{T} R K_{d \tau} \\
& +Z_{\tau}-S_{\tau} \leq 0 .
\end{aligned}
$$

Multiplying $W_{\tau}^{T}$ and $\gamma^{-1} W_{\tau}$ in both sides of inequality (40), then

$$
\begin{aligned}
(2 l+2) & \left(B K_{d \tau} W_{\tau}\right)^{T}\left(\gamma P^{-1}-\gamma \varepsilon_{\tau} H H^{T}\right)^{-1}\left(B K_{d \tau} W_{\tau}\right) \\
& +(2 l+2) \gamma^{-1} \varepsilon_{\tau}^{-1}\left(E_{2} K_{d \tau} W_{\tau}\right)^{T}\left(E_{2} K_{d \tau} W_{\tau}\right) \\
& +(l+1)\left(K_{d \tau} W_{\tau}\right)^{T} \gamma^{-1} R\left(K_{d \tau} W_{\tau}\right) \\
& +W_{\tau}^{T} \gamma^{-1} Z_{\tau} W_{\tau}-W_{\tau}^{T} \gamma^{-1} S_{\tau} W_{\tau} \leq 0 .
\end{aligned}
$$

Since $P^{-1}=\gamma^{-1} Q, S_{1}^{-1}=\gamma^{-1} W_{1}, \ldots, S_{l}^{-1}=\gamma^{-1} W_{l}$, and choose $\Lambda_{\tau}=\gamma \varepsilon_{\tau}, K_{d \tau} W_{\tau}=Y_{\tau}, Z_{\tau}=\gamma M_{\tau}{ }^{-1}$; then by using Schur complement [19], inequality (41) can be derived as the following inequalities:

$$
\begin{array}{ccccc}
W_{\tau} & * & * & * & * \\
R^{0.5} Y_{\tau} & \frac{1}{l+1} \gamma & * & * & * \\
E_{2} Y_{\tau} & 0 & \frac{1}{2 l+2} \Lambda_{\tau} & * & * \\
W_{\tau} & 0 & 0 & M_{\tau} & * \\
B Y_{\tau} & 0 & 0 & 0 & \frac{1}{2 l+2}\left(Q-\Lambda_{\tau} H H^{T}\right) \\
>0 & & {\left[\begin{array}{cc}
Q & * \\
H^{T} & \Lambda_{\tau}^{-1}
\end{array}\right]>0,}
\end{array}
$$

where $\tau=1, \ldots, l$.

Multiplying both sides by $\gamma^{-1}$ and using Schur complement, $(2 l+2) A_{d \tau}^{T} P A_{d \tau}-Z_{\tau} \leq 0$ can be derived as follows:

$$
\left[\begin{array}{cc}
M_{\tau} & * \\
A_{d \tau}^{T} & \frac{1}{2 l+2} Q
\end{array}\right]>0
$$

where $\tau=1, \ldots, l$.

Secondly, we convert the input constraint (2) into LMI form. The upper bound of the object function (14) can be rewritten as

$$
\begin{aligned}
& \|x(k+i \mid k)\|_{P}+\sum_{\tau=1}^{l} \sum_{j=1}^{\tilde{d}_{\tau}}\|x(k+i-j \mid k)\|_{S_{\tau}} \\
& +\sum_{\tau=1}^{l} \sum_{j=\tilde{d}_{\tau}+1}^{\tilde{d}_{\tau}+\eta_{\tau}}\|x(k+i-j \mid k)\|_{Z_{\tau}} \leq \gamma(k) .
\end{aligned}
$$


Since $\gamma>0$, then inequality (44) is equivalent to

$$
\begin{aligned}
& \|x(k+i \mid k)\|_{P} \\
& +\left[\sum_{j=1}^{\tilde{d}_{1}}\|x(k+i-j \mid k)\|_{S_{1}}+\cdots+\sum_{j=1}^{\tilde{d}_{l}}\|x(k+i-j \mid k)\|_{S_{l}}\right] \\
& +\left[\sum_{j=\tilde{d}_{1}+1}^{\widetilde{d}_{1}+\eta_{1}}\|x(k+i-j \mid k)\|_{Z_{1}}\right. \\
& \left.+\cdots+\sum_{j=\widetilde{d}_{l}+1}^{\tilde{d}_{l}+\eta_{l}}\|x(k+i-j \mid k)\|_{Z_{l}}\right]
\end{aligned}
$$$$
\leq \gamma(k)
$$

and then inequality (45) can be rewritten as follows:

$$
\begin{gathered}
\|x(k+i \mid k)\|_{\gamma^{-1} P}+\sum_{j=1}^{\tilde{d}_{1}}\|x(k+i-j \mid k)\|_{\gamma^{-1} S_{1}} \\
+\sum_{j=\widetilde{d}_{1}+1}^{\widetilde{d}_{1}+\eta_{1}}\|x(k+i-j \mid k)\|_{\gamma^{-1} Z_{1}} \\
+\cdots+\sum_{j=1}^{\tilde{d}_{l}}\|x(k+i-j \mid k)\|_{\gamma^{-1} S_{l}} \\
+\sum_{j=\widetilde{d}_{l}+1}^{\tilde{d}_{l}+\eta_{l}}\|x(k+i-j \mid k)\|_{\gamma^{-1} Z_{l}} \leq 1 .
\end{gathered}
$$

For $Q=\gamma P^{-1}>0, W_{1}=\gamma S_{1}^{-1}>0, \ldots, W_{l}=\gamma S_{l}^{-1}>0$, $M_{1}=\gamma Z_{1}^{-1}>0, \ldots, M_{l}=\gamma Z_{l}^{-1}>0$, then we obtain that

$$
\begin{gathered}
\|x(k+i \mid k)\|_{Q}+\sum_{j=1}^{\tilde{d}_{1}}\|x(k+i-j \mid k)\|_{W_{1}} \\
+\sum_{j=\widetilde{d}_{1}+1}^{\tilde{d}_{1}+\eta_{1}}\|x(k+i-j \mid k)\|_{M_{1}} \\
+\cdots+\sum_{j=1}^{\tilde{d}_{l}}\|x(k+i-j \mid k)\|_{W_{l}} \\
+\sum_{j=\widetilde{d}_{l}+1}^{\tilde{d}_{l}+\eta_{l}}\|x(k+i-j \mid k)\|_{M_{l}} \leq 1 .
\end{gathered}
$$

Moreover, $Q>0, W_{\tau}>0$, and $M_{\tau}>0$; we can obtain that

$$
\begin{gathered}
x^{T}(k+i \mid k) Q x(k+i \mid k)<1 \\
x^{T}\left(k+i-\widetilde{d}_{1} \mid k\right) W_{1} x\left(k+i-\widetilde{d}_{1} \mid k\right)<1 \\
\vdots \\
x^{T}\left(k+i-\tilde{d}_{l} \mid k\right) W_{l} x\left(k+i-\tilde{d}_{l} \mid k\right)<1 \\
x^{T}\left(k+i-\tilde{d}_{1}-\eta_{1} \mid k\right) M_{1} x\left(k+i-\tilde{d}_{1}-\eta_{1} \mid k\right)<1 \\
\vdots \\
x^{T}\left(k+i-\tilde{d}_{l}-\eta_{l} \mid k\right) M_{l} x\left(k+i-\tilde{d}_{l}-\eta_{l} \mid k\right)<1 .
\end{gathered}
$$

From (7), $K=Y Q^{-1}$, and $K_{d \tau}=Y_{\tau} W_{\tau}^{-1}(\tau=1, \ldots, l)$, the input constraint can be expressed as

$$
\begin{aligned}
& \|u(k+i \mid k)\|_{2} \\
& \leq u_{\max } \\
& \Longleftrightarrow\left\|K x(k+i \mid k)+\sum_{\tau=1}^{l} K_{d \tau} x\left(k+i-\tilde{d}_{\tau} \mid k\right)\right\|_{2} \leq u_{\max } \\
& \Longleftarrow\|K x(k+i \mid k)\|_{2}+\left\|K_{d 1} x\left(k+i-\tilde{d}_{1} \mid k\right)\right\|_{2} \\
& \quad+\cdots+\left\|K_{d l} x\left(k+i-\tilde{d}_{l} \mid k\right)\right\|_{2} \leq u_{\max } \\
& \Longleftrightarrow\left\|Y Q^{-1} x(k+i \mid k)\right\|_{2}+\left\|Y_{1} W_{1}^{-1} x\left(k+i-\tilde{d}_{1} \mid k\right)\right\|_{2} \\
& \quad+\cdots+\left\|Y_{l} W_{l}^{-1} x\left(k+i-\tilde{d}_{l} \mid k\right)\right\|_{2} \leq u_{\max } .
\end{aligned}
$$

Inequality (49) is satisfied if

$$
\left[\begin{array}{ccccc}
u_{\max }^{2} I & * & * & * & * \\
Y^{T} & Q & * & * & * \\
Y_{1}^{T} & 0 & W_{1} & * & * \\
\vdots & \vdots & 0 & \ddots & * \\
Y_{l}^{T} & 0 & \cdots & 0 & W_{l}
\end{array}\right] \geq 0
$$

holds. This completes the proof.

Now, the minimization problem (15) is converted to the following LMI optimization problem:

$$
\min _{\gamma(k), P, W_{1}, \ldots, W_{l}, Y, Y_{1}, \ldots, Y_{l}} \gamma(k)
$$$$
\text { subject to (16) and (17). }
$$

Theorem 4 (closed-loop stability). If the optimization problem (51) is feasible at the initial instant $k=0$, then the proposed robust memory state feedback MPC law (7) stabilizes system (1) asymptotically. 
Proof. Let $P(k)$ and $P(k+1)$ denote the optimal solutions of the optimization problem (51) at instant $k$ and $k+1$, respectively. Let us consider a quadratic function

$$
\begin{aligned}
V(x(k))= & \|x(k)\|_{P(k)}+\sum_{\tau=1}^{l} \sum_{j=1}^{\tilde{d}_{\tau}}\|x(k-j)\|_{S_{\tau}} \\
& +\sum_{\tau=1}^{l} \sum_{j=\widetilde{d}_{\tau}+1}^{\widetilde{d}_{\tau}+\eta_{\tau}}\|x(k-j \mid k)\|_{Z_{\tau}} .
\end{aligned}
$$

Since $P(k+1)$ is optimal while $P(k)$ is only feasible at instant $k+1$,

$$
\begin{aligned}
V(x(k+1))= & \|x(k+1)\|_{P(k+1)}+\sum_{\tau=1}^{l} \sum_{j=1}^{\tilde{d}_{\tau}}\|x(k-j+1)\|_{S_{\tau}} \\
& +\sum_{\tau=1}^{l} \sum_{j=\tilde{d}_{\tau}+1}^{\tilde{d}_{\tau}+\eta_{\tau}}\|x(k-j+1 \mid k)\|_{Z_{\tau}} \\
\leq & \|x(k+1)\|_{P(k)}+\sum_{\tau=1}^{l} \sum_{j=1}^{\tilde{d}_{\tau}}\|x(k-j+1)\|_{S_{\tau}} \\
& +\sum_{\tau=1}^{l} \sum_{j=\tilde{d}_{\tau}+1}^{\tilde{d}_{\tau}+\eta_{\tau}}\|x(k-j+1 \mid k)\|_{Z_{\tau}} .
\end{aligned}
$$

Besides, it follows from (11) that

$$
\begin{aligned}
\|x(k+1 \mid k)\|_{P(k)}+\sum_{\tau=1}^{l} \sum_{j=1}^{\tilde{d}_{\tau}}\|x(k-j+1 \mid k)\|_{S_{\tau}} \\
+\sum_{\tau=1}^{l} \sum_{j=\widetilde{d}_{\tau}+1}^{\tilde{d}_{\tau}+\eta_{\tau}}\|x(k-j+1 \mid k)\|_{Z_{\tau}} \\
\leq\|x(k \mid k)\|_{P(k)}+\sum_{\tau=1}^{l} \sum_{j=1}^{\tilde{d}_{\tau}}\|x(k-j \mid k)\|_{S_{\tau}} \\
+\sum_{\tau=1}^{l} \sum_{j=\tilde{d}_{\tau}+1}^{\tilde{d}_{\tau}+\eta_{\tau}}\|x(k-j \mid k)\|_{Z_{\tau}} \\
\quad-\left[\|x(k \mid k)\|_{Q_{1}}+\|u(k \mid k)\|_{R}\right]
\end{aligned}
$$

for any structured uncertainties $[\Delta A, \Delta B]$.

Since $x(k+1 \mid k+1)$ equals $A(k) x(k \mid k)+\sum_{\tau=1}^{l} A_{d \tau} x(k-$ $\left.d_{\tau} \mid k\right)+B(k) u(k \mid k)$ for given uncertainties, inequality (54) must hold true with $x(k+1 \mid k+1)$ in place of $x(k+1 \mid k)$. Combining this with (53), we have

$$
\begin{aligned}
& V(x(k+1 \mid k+1)) \\
& \quad \leq V(x(k \mid k))-\left[\|x(k \mid k)\|_{\mathrm{Q}_{1}}+\|u(k \mid k)\|_{R}\right] .
\end{aligned}
$$

Summing inequality (55) from $k=0$ to $k=i-1$ yields

$$
V(x(i \mid i))+\sum_{k=0}^{i-1}\left[\|x(k \mid k)\|_{Q_{1}}+\|u(k \mid k)\|_{R}\right] \leq V(x(0 \mid 0)) \text {. }
$$

Since $V(x(i \mid i)) \geq 0$ and the left hand side of (56) is bounded above by the constant $V(x(0 \mid 0)), x(i \mid i)$ and $u(i \mid i)$ must go to zero as $i$ goes to infinity. Therefore, the closed-loop stability is guaranteed.

\section{Numerical Example}

In this section, a numerical example is presented to illustrate the effectiveness of the proposed delay-dependent feedback robust MPC algorithm. Let us consider the following discrete-time multiple time-delayed linear uncertain system:

$$
x(k+1)=A(k) x(k)+\sum_{\tau=1}^{l} A_{d \tau} x\left(k-d_{\tau}\right)+B(k) u(k),
$$

where $A=\left[\begin{array}{cc}0.0 & 1.2 \\ 1.0 & -1.5\end{array}\right], A_{d 1}=\left[\begin{array}{ll}0.2 & 0.2 \\ 0.2 & 0.1\end{array}\right], A_{d 2}=\left[\begin{array}{ll}0.02 & 0.02 \\ 0.02 & 0.01\end{array}\right], B=$ $\left[\begin{array}{ll}1.1 & 1.3\end{array}\right]^{T}, E_{1}=\left[\begin{array}{ll}0.2 & 0.3\end{array}\right], E_{2}=0.9, F=\sin k$, and $H=$ $\left[\begin{array}{ll}0.1 & 0.1\end{array}\right]^{T}$.

The initial value state is given as $x(0)=[1,1]^{T}$, and the state and the input weighting matrices are $Q_{1}=\operatorname{diag}(1,1)$ and $R=0.05$. Assume that the time delay index are $d_{1}=2$ and $d_{2}=6$, and $l=2$. Assume that the estimated time-delay indexes are $\tilde{d}_{1}=2, \widetilde{d}_{2}=5$; then the estimated errors are $\eta_{1}=$ $1, \eta_{2}=1$, respectively. And the control constraint is $|u| \leq 2$.

The controllers are listed as follows:

(i) $u(k+i \mid k)=K(k) x(k+i \mid k)$; the controller is referred to as a memoryless controller obtained by Kothare's method;

(ii) $u(k+i \mid k)=K(k) x(k+i \mid k)+K_{d 1}(k) x\left(k+i-d_{1} \mid\right.$ $k)+K_{d 2}(k) x\left(k+i-d_{2} \mid k\right)$; the controller is referred to as a multiple time-delayed exact-memory controller with time-delayed state $x\left(k-d_{1} \mid k\right)$ and $x\left(k-d_{2} \mid k\right)$ in this paper;

(iii) $u(k+i \mid k)=K(k) x(k+i \mid k)+\sum_{\tau=1}^{l} K_{d \tau}(k) x\left(k+i-\widetilde{d}_{\tau} \mid\right.$ $k)$; the controller is referred to as a memory controller with estimated delayed state indices in this paper.

The simulation results are compared with the results that were obtained by Kothare's method with a memoryless state feedback controller in [4] and the results that were obtained by the method in [15] with a memory-resilient state feedback controller. In Figure 1, the stabilization performances for the three techniques are also compared. From Figure 2, the inputs are all within their limits and no saturation is observed all the time. Moreover, Figure 3 shows that the cost function by the memory controller with estimated delayed states is smaller than the one obtained by the technique of a memoryless state feedback controller but is larger than the one obtained by the technique of the exact-memory controller. We can also 


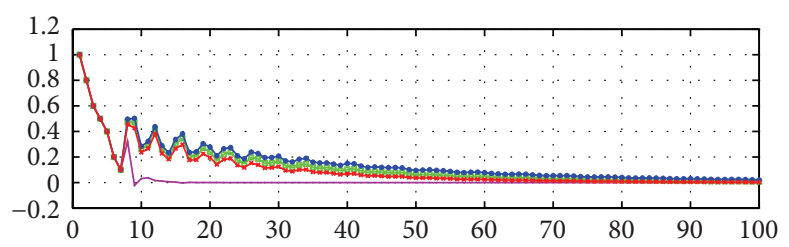

_ The multiple delayed state exact-memory controller in this paper

- The memory-resilient controller obtained by the method in [15]

- The memoryless controller obtained by Kothare's method

— The multiple delayed state memory feedback controller with estimated delayed states in this paper

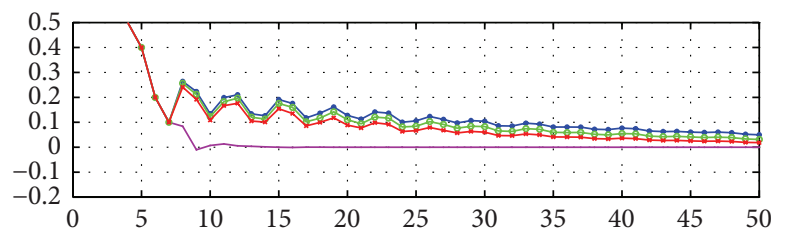

- The multiple delayed state exact-memory controller in this paper

- The memoryless controller obtained by Kothare's method

$\rightarrow$ The memory-resilient controller obtained by the method in [15]

- The multiple delayed state memory feedback controller with estimated delayed states in this paper

FIGURE 1: State responses for $x_{1}$ and $x_{2}$.

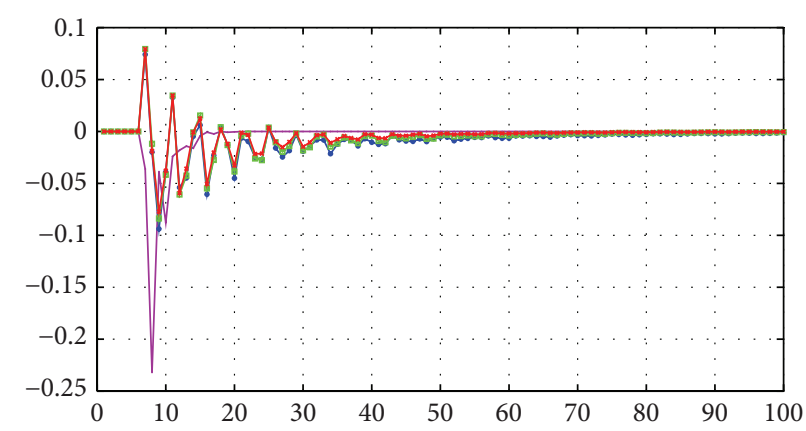

_ The multiple delayed state exact-memory controller in this paper

$\rightarrow$ The memoryless controller obtained by Kothare's method

$\rightarrow$ The memory-resilient controller obtained by the method in [15]

—- The multiple delayed state memory feedback controller with estimated delayed states in this paper

Figure 2: Control input.

obtain that the cost function obtained by the controller in this paper is smaller than the one obtained by the technique of [15], and the cost function obtained by the technique of [15] is smaller than the memoryless state feedback controller. We can also see that the improvement is more evident in Figures 1 and 3 . In the simulation results, stabilization performance as well as the cost is improved by using the memory state feedback RMPC. The above discussion illustrates that the memory controllers with the estimated time-delayed states define the intermediary behavior of the closed-loop system between the two external controllers: the memoryless and the exact-memory controllers. Moreover, such controller with

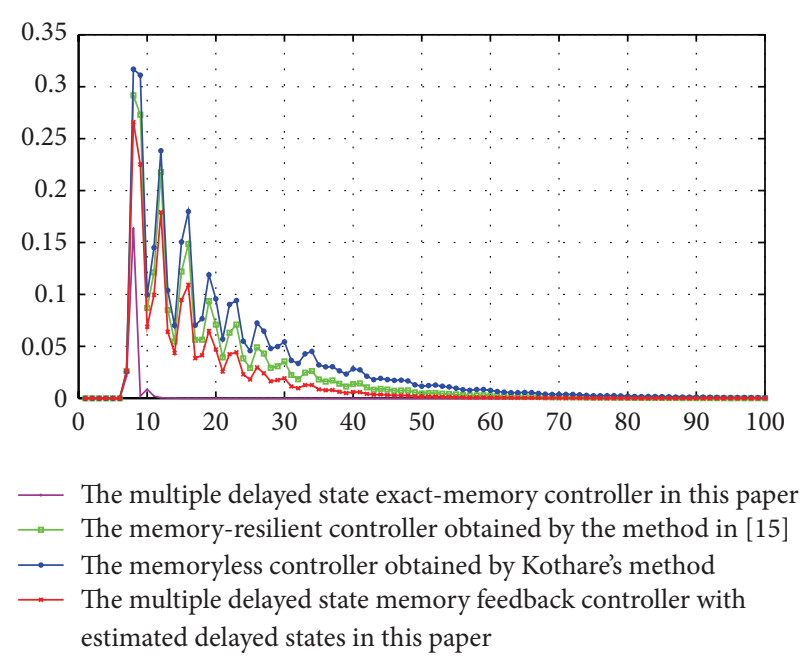

FIgURE 3: Cost index.

the estimated time-delayed state indices is also more realistic, from a practical point of view, than the exact-memory controllers.

\section{Conclusions}

In this paper, we have proposed a new delay-dependent feedback robust MPC technique for a class of multiple uncertain time-delayed linear uncertain systems with input constraints. Compared with other works in the literature, the main novelty of the proposed approach was taking the information of the multiple delayed states with the estimated time-delays indices into full consideration, by choosing a delayed state dependent Lyapunov function. The minimization problem for infinite horizon cost was derived using estimated delayed state indices. Since the proposed approach allows for the synthesis of delay-dependent robust controllers with respect to uncertainties on the implemented delay, the new improved method is much less conservative and more general from a practical point of view. Numerical examples demonstrated the effectiveness of the proposed method.

\section{Conflict of Interests}

The authors declare that there is no conflict of interests regarding the publication of this paper.

\section{Acknowledgments}

This work is supported in part by the National Natural Science Foundation (NNSF) of China under Grant nos. 61304001 and 61403399. The authors would also like to thank Xiaoxiang HU and Yang GUO for Matlab simulations.

\section{References}

[1] D. Q. Mayne, J. B. Rawlings, C. V. Rao, and P. O. Scokaert, "Constrained model predictive control: stability and optimality," Automatica, vol. 36, no. 6, pp. 789-814, 2000. 
[2] Y. G. Xi and D. W. Li, "Fundamental philosophy and status of qualitative synthesis of model predictive control," Acta Automatica Sinica, vol. 34, no. 10, pp. 1225-1234, 2008.

[3] Y. G. Xi, D. W. Li, and S. Lin, "Model predictive control—current status and challenges," Acta Automatica Sinica, vol. 39, no. 3, pp. 222-236, 2013.

[4] M. V. Kothare, V. Balakrishnan, and M. Morari, "Robust constrained model predictive control using linear matrix inequalities," Automatica, vol. 32, no. 10, pp. 1361-1379, 1996.

[5] W. H. Kwon, J. W. Kang, Y. S. Lee, and Y. S. Moon, "A simple receding horizon control for state delayed systems and its stability criterion," Journal of Process Control, vol. 13, no. 6, pp. 539-551, 2003.

[6] J.-C. Zhao, Y.-M. Fang, and J.-X. Li, "Robust predictive control for a class of uncertain discrete system with multiple state delays," in Proceedings of the 30th Chinese Control Conference (CCC '11), pp. 3372-3376, Yantai, China, July 2011.

[7] W.-W. Qin, G. Liu, L.-X. Wang, and Z.-Q. Zheng, "Memory state feedback RMPC for multiple time-delayed uncertain linear systems with input constraints," Mathematical Problems in Engineering, vol. 2014, Article ID 409863, 9 pages, 2014.

[8] S. C. Jeong and P. G. Park, "Constrained MPC algorithm for uncertain time-varying systems with state-delay," IEEE Transactions on Automatic Control, vol. 50, no. 2, pp. 257-263, 2005.

[9] L. Mei and S. Huihe, "Robust predictive control of polytopic uncertain systems with both state and input delays," Journal of Systems Engineering and Electronics, vol. 18, no. 3, pp. 616-621, 2007.

[10] F. Gouaisbaut and D. Peaucelle, "Delay-dependent robust stability of time delay systems," in Proceedings of the 5th IFAC Symposium on Robust Control Design (ROCOND '06), pp. 453458, Toulouse, France, July 2006.

[11] C. Y. Kao and A. Rantzer, "Stability analysis of systems with uncertain time-varying delays," Automatica, vol. 43, no. 6, pp. 959-970, 2007.

[12] D. H. Ji, J. H. Park, W. J. Yoo, and S. C. Won, "Robust memory state feedback model predictive control for discrete-time uncertain state delayed systems," Applied Mathematics and Computation, vol. 215, no. 6, pp. 2035-2044, 2009.

[13] C.-Y. Chen and C.-H. Lee, "Delay-independent stabilization of linear systems with time-varying delayed state and uncertainties," Journal of the Franklin Institute, vol. 346, no. 4, pp. 378390, 2009.

[14] W. W. Qin, G. Liu, and Z. Q. Zheng, "Memory state feedbackbased model predictive controller of time-delay systems with input constraints," Journal of South China University of Technology, vol. 40, no. 6, pp. 63-69, 2012.

[15] C. Briat, O. Sename, and J. F. Lafay, "Memory-resilient gainscheduled state-feedback control of uncertain LTI/LPV systems with time-varying delays," Systems \& Control Letters, vol. 59, no. 8, pp. 451-459, 2010.

[16] P.-L. Liu, "State feedback stabilization of time-varying delay uncertain systems: a delay decomposition approach," Linear Algebra and Its Applications, vol. 438, no. 5, pp. 2188-2209, 2013.

[17] C. L. Su, J. C. Zhao, and P. Li, "Robust predictive control for a class of multiple time delay uncertain systems with nonlinear disturbance," Acta Automatica Sinica, vol. 39, no. 5, pp. 644-649, 2013.

[18] Z. D. Wang, B. Huang, and H. Unbehauen, "Robust reliable control for a class of uncertain nonlinear state-delayed systems," Automatica, vol. 35, no. 5, pp. 955-963, 1999.
[19] S. Boyd, L. E. Ghaoui, E. Feron, and V. Balakrishnan, Linear Matrix Inequalities in System and Control Theory, SIAM, Philadelphia, Pa, USA, 1994. 


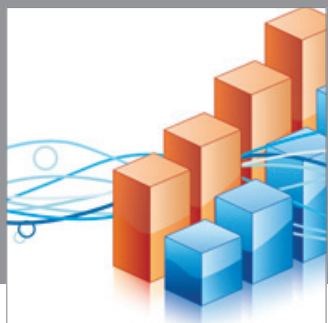

Advances in

Operations Research

mansans

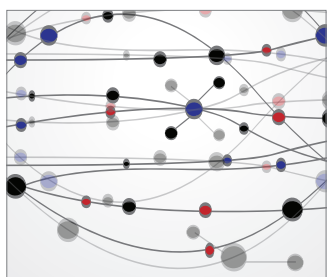

The Scientific World Journal
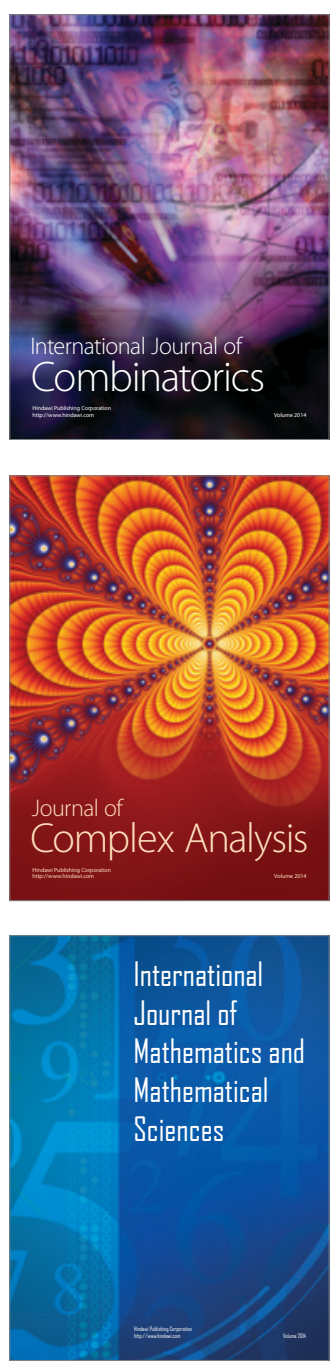
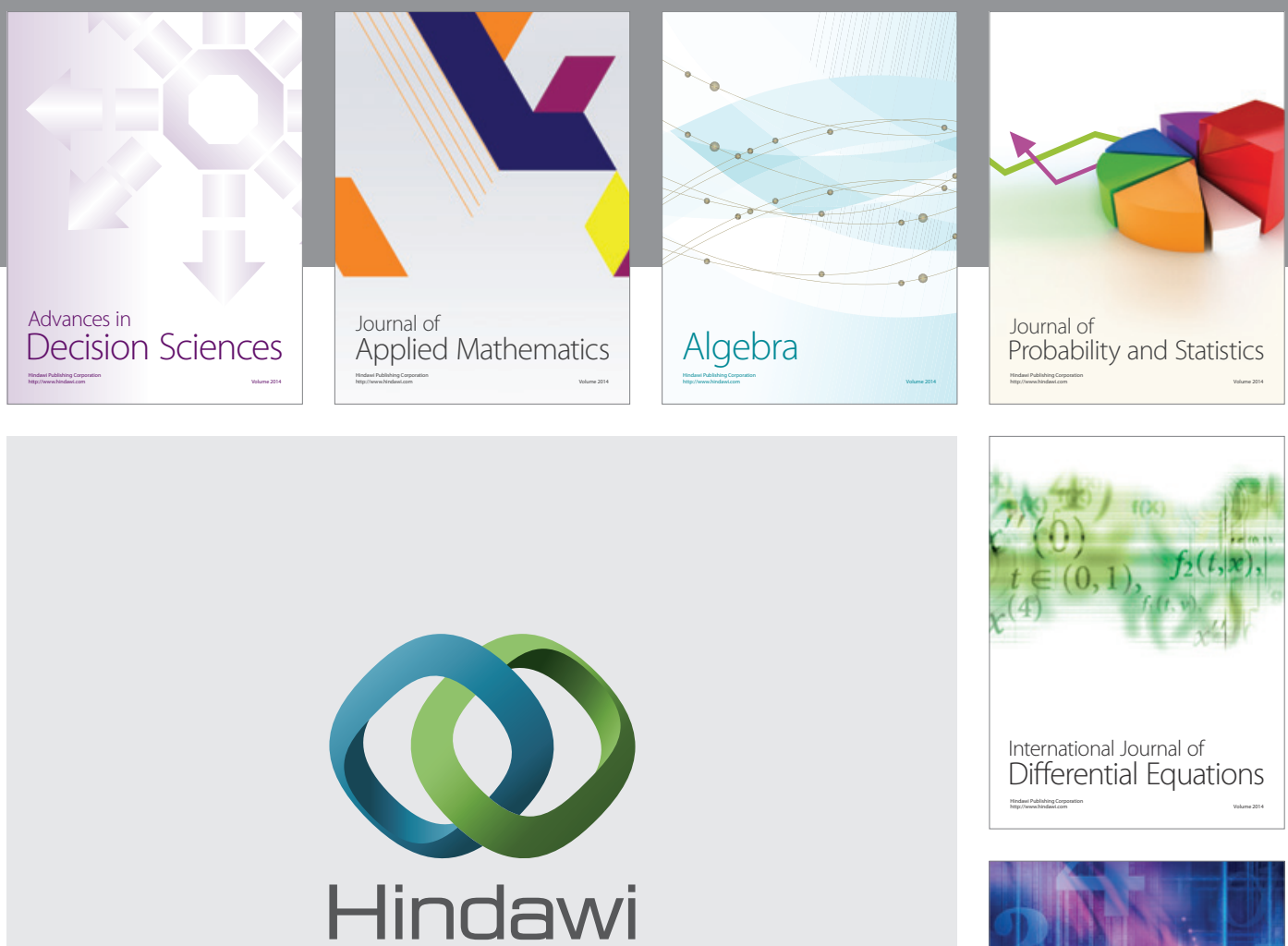

Submit your manuscripts at http://www.hindawi.com
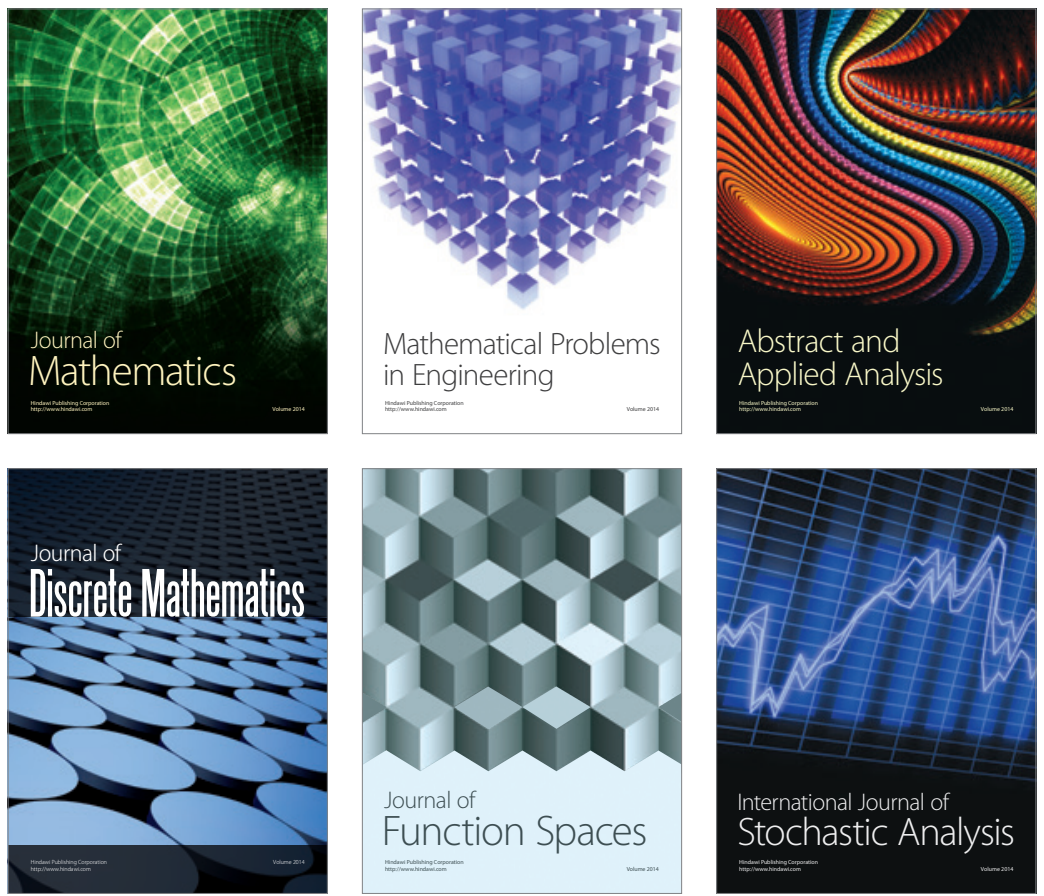

Journal of

Function Spaces

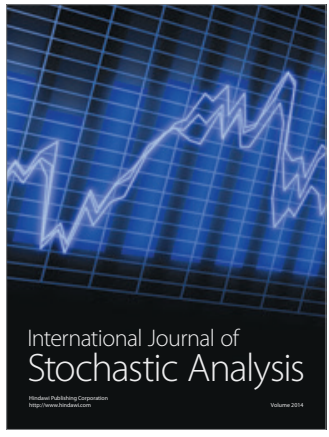

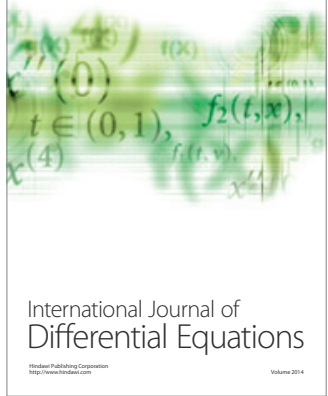
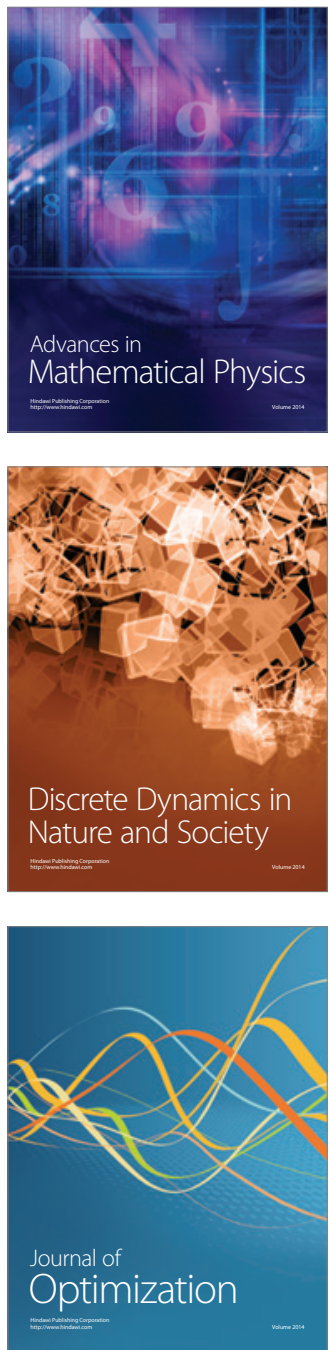